\title{
Double-Decker Silsesquioxanes Self-Assembled in One-Dimensional Coordination Polymeric Nanofibers with Emission Properties
}

Julia Duszczak, ${ }^{\ddagger[\mathrm{a}]}$ Katarzyna Mituła, ${ }^{\ddagger[\mathrm{a}]}$ Andrea Santiago-Portillo, ${ }^{*[\mathrm{~b}]}$ Loraine Soumoy, ${ }^{[\mathrm{b}]}$ Monika Rzonsowska, ${ }^{[\mathrm{a}]}$ Rafał Januszewski, ${ }^{[\mathrm{c}]}$ Luca Fusaro, ${ }^{[\mathrm{b}]}$ Carmela Aprile, ${ }^{*[\mathrm{~b}]}$ and Beata Dudziec ${ }^{*[\mathrm{a}]}$

${ }^{[a]}$ Department of Organometallic Chemistry, Faculty of Chemistry, Centre for Advanced Technologies, Adam Mickiewicz University in Poznan, Uniwersytetu Poznanskiego 8 and 10, 61-614 Poznan, Poland.

[b] Department of Chemistry, University of Namur, Rue de Bruxelles 61, 5000 Namur, Belgium.

${ }^{[c]}$ Department of Chemistry and Technology of Silicon Compounds, Faculty of Chemistry, Centre for Advanced Technologies, Adam Mickiewicz University in Poznan, Uniwersytetu Poznanskiego 8 and 10, 61-614 Poznan, Poland.

*E-mail:beata.dudziec@gmail.com,carmela.aprile@unamur.be,andrea.santiago@unamur.be

$\ddagger$ Julia Duszczak and Katarzyna Mituła contributed equally.

Table of contents:

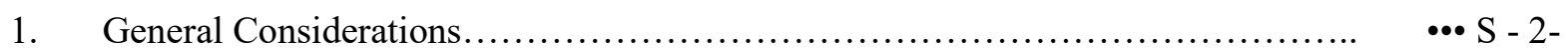

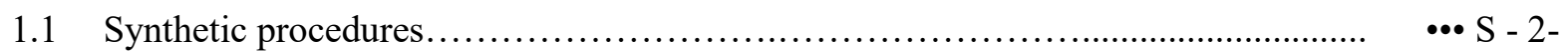

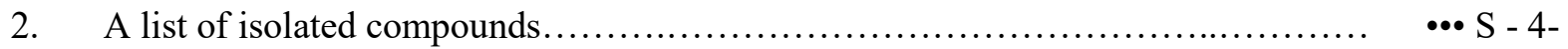

3. Analytical data of obtained compounds.................................................. $\mathrm{S}-5-$

3.1 Thermal analysis of reagents DDSQa-b and DDSQ_Ta-b.......................... $\mathrm{S}-16-$

4. Results of absorption - emission analysis of DDSQa-b, DDSQ_Ta-b and metal@DDSQ-based complexes.....

5. Results of Transmission and Scanning Electron Microscopy of DDSQa-b, DDSQ_Ta-b and and metal@DDSQ-based complexes...

6. References 


\section{General Considerations}

\subsection{Synthetic procedures}

General synthetic procedure for the synthesis of DDSQa-b via silylative coupling reaction

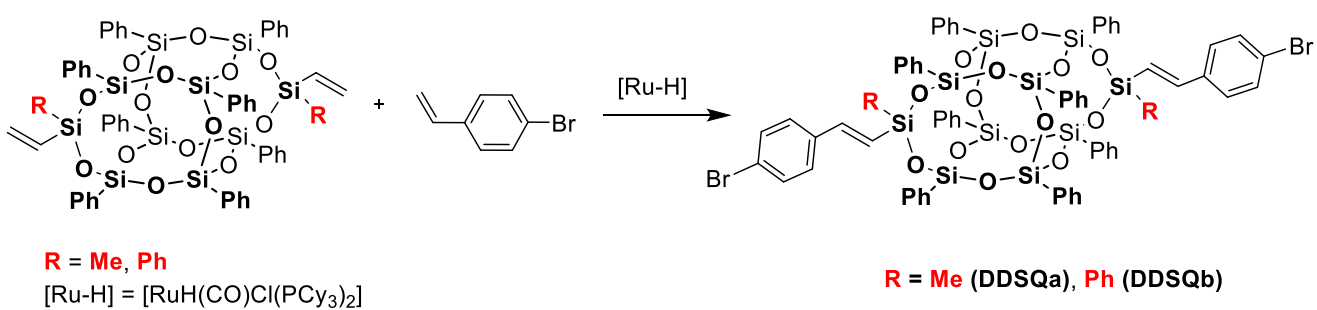

Scheme S1. Synthesis of DDSQa-b via silylative coupling reaction.

The respective synthetic protocol is presented for DDSQa and is analogous for DDSQb.

The glass Schlenk reactor equipped with a magnetic stirring bar was evacuated and flushed with argon, a $\left[\mathrm{RuH}(\mathrm{CO}) \mathrm{Cl}\left(\mathrm{PCy}_{3}\right)_{2}\right]\left(1.21 \mathrm{mg}, 1.662 \times 10^{-6} \mathrm{mmol}\right)$ and toluene $(0.66 \mathrm{~mL})$, then DDSQ-2(MeSiVi) $(100 \mathrm{mg}$, $0.0831 \mathrm{mmol})$ and 4-bromostyrene $(0.243 \mathrm{mmol}, 35 \mu \mathrm{L})$ were added respectively. The reaction was conducted in a closed system for $48 \mathrm{~h}$ at $120^{\circ} \mathrm{C}$. After the reaction was completed, the solvent and any excess of volatile reagents were removed under reduced pressure. The crude product (DDSQa) was filtered off by column chromatography (silica gel 60, hexane and dichloromethane 1:5) to remove the catalyst, then precipitated in methanol as white solid.

\section{Synthesis of 4'-(4-Ethylnylphenyl)-[2,2':6,2'’]terpyridine -T}

This compound was synthesized with small changes as previously reported. ${ }^{1}$ In this case, $7.68 \mathrm{mmol}$ of $\mathrm{NaOH}$ was added to a round-bottom flask with $10 \mathrm{~mL}$ of PEG 300 at $0{ }^{\circ} \mathrm{C}$. After that, $7.68 \mathrm{mmol}$ of 2-acetylpyridine was added. After $10 \mathrm{~min}$ of magnetic stirred, $3.38 \mathrm{mmol}$ of 4-ethynylbenzaldehyde was added to the solution and it was stirred at $0{ }^{\circ} \mathrm{C}$ for $4 \mathrm{~h}$ (instead of reported $2 \mathrm{~h}$ ). Then, $10 \mathrm{~mL}$ of concentrated ammonia aqueous solution was added and the suspension was stirred at room temperature overnight (instead of reported $2 \mathrm{~h}$ ). Subsequently, the solid was isolated by filtration and washed several times with water and cold ethanol. Extension of reaction time resulted in obtaining better isolation yield (70\% instead of reported $44 \%)$.

\section{General synthetic procedure for the synthesis of DDSQ_Ta-b via Sonogashira reaction}

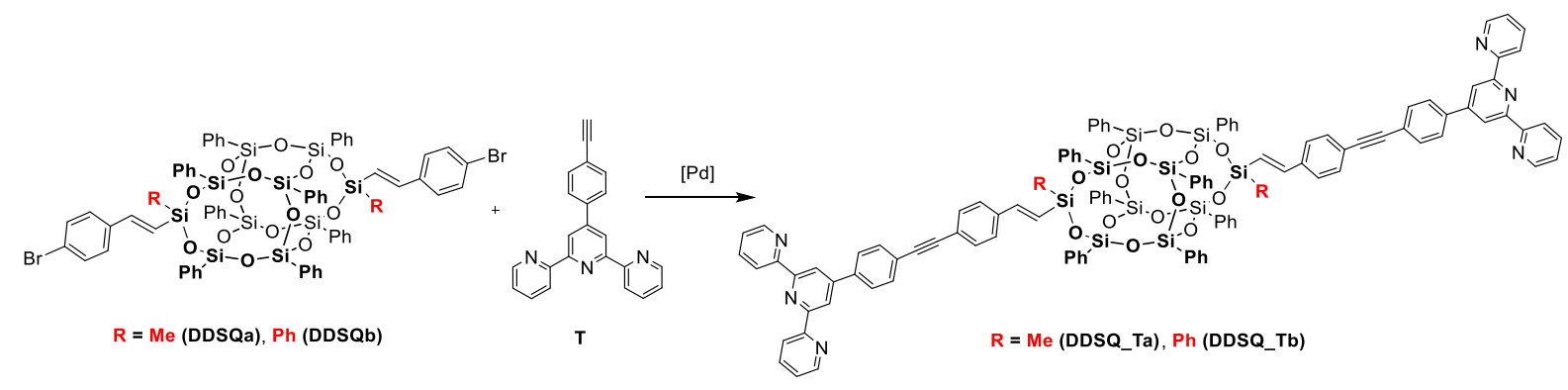

Scheme S2. Synthesis of DDSQ_Ta-b via Sonogashira reaction.

The exemplary procedure is presented for the DDSQ_Ta compound and is analogous for DDSQ_Tb.

Into a glass Schlenk reactor equipped with a magnetic stirring bar, DDSQa (200 mg, $0.122 \mathrm{mmol}), \mathrm{T}$ (122 mg, $0.366 \mathrm{mmol}), \mathrm{Pd}\left(\mathrm{PPh}_{3}\right)_{4}(51 \mathrm{mg}, 0.044 \mathrm{mmol})$ and $\mathrm{CuI}(9 \mathrm{mg} 0.049 \mathrm{mmol})$ were added respectively. Then the reactor was flushed with nitrogen for 30 minutes. Afterward dry THF (15 mL) and $\mathrm{Et}_{3} \mathrm{~N}$ or (iPr) $)_{2} \mathrm{NH}(5 \mathrm{~mL})$ were added in the presence of nitrogen/argon. The reaction was conducted in a closed system for $24 \mathrm{~h}$ at $70{ }^{\circ} \mathrm{C}$. Then the mixture was filtered from insoluble solids and concentrated in vacuo. The crude product (DDSQa) was washed with EtOH and water and then several times $(5 \times 15 \mathrm{~mL})$ with EtOH using the ultrasound bath for 10 min and centrifuged $\left(10{ }^{\circ} \mathrm{C}, 4500 \mathrm{rpm}, 15 \mathrm{~min}\right.$.) receiving brown solid. 


\section{General synthetic protocols of preparation of metal@DDSQ-based complexes}

The exemplary procedure is presented for Fe@DDSQ_Ta.

DDSQ_Ta $(3 \mathrm{mg})$ and $10 \mathrm{~mL}$ of $\mathrm{CH}_{2} \mathrm{Cl}_{2}$ were placed into a glass vial and stirred vigorously using the ultrasound bath for $10 \mathrm{~min}\left(1.485 \times 10^{-4} \mathrm{M}\right.$ solution). A solution of $\mathrm{Fe}(\mathrm{OTf})_{2}(3 \mathrm{mg}, 85 \%$ purity) and $20 \mathrm{~mL}$ of EtOH was prepared in another vial, also using an ultrasound bath for $10 \mathrm{~min}\left(3.63 \times 10^{-4} \mathrm{M}\right.$ solution). Afterward, $67 \mu \mathrm{L}$ of prepared DDSQ_Ta in $\mathrm{CH}_{2} \mathrm{Cl}_{2}$ was diluted with $9.783 \mathrm{~mL}$ of $\mathrm{CH}_{2} \mathrm{Cl}_{2}$, to obtain $1 \times 10^{-6} \mathrm{M}$ concentration. DDSQ_Ta in $\mathrm{CH}_{2} \mathrm{Cl}_{2}$ was subjected to titration with $5 \mu \mathrm{L}$ portions of $\mathrm{Fe}(\mathrm{OTf})_{2}$ in EtOH, with 20 min. of vigorous stirring after each addition. The titration was conducted until the equilibrium was reached.

\section{General synthetic protocols of preparation of solids metal@DDSQ-based complexes}

The exemplary procedure is presented for Fe@DDSQ_Ta.

DDSQ_Ta $\left(15 \mathrm{mg}, 7.42 \times 10^{-3} \mathrm{mmol}\right)$ and $6 \mathrm{~mL}$ of $\mathrm{CH}_{2} \mathrm{Cl}_{2}$ were placed into a glass vial and stirred vigorously using the ultrasound bath for $10 \mathrm{~min}$. A solution of $\mathrm{Fe}(\mathrm{OTf})_{2}\left(3.1 \mathrm{mg}, 7.42 \times 10^{-3} \mathrm{mmol} 85 \%\right.$ purity) and $4 \mathrm{~mL}$ of EtOH was prepared in another vial, also using an ultrasound bath for $10 \mathrm{~min}$. After that, the solution of $\mathrm{Fe}(\mathrm{OTf})_{2}$ was placed drop by drop to the vial that contains DDSQ_Ta. The mixture was left at room temperature until it was almost dry. Afterward, the sample was left in the freezer and then it was dried in a freeze dryer. 
2. Table S1. A list of isolated compounds

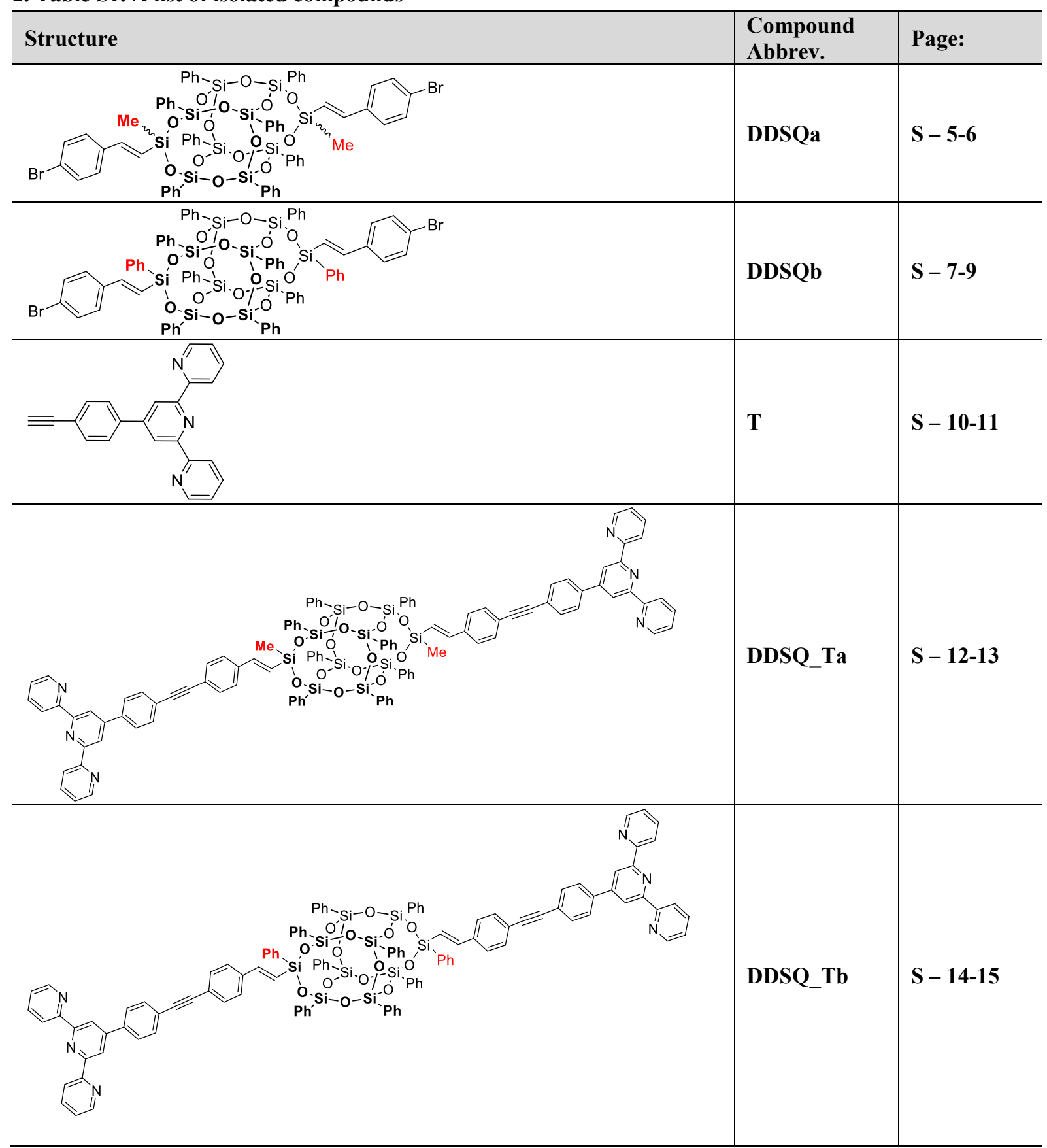




\section{Analytical data of obtained compounds}

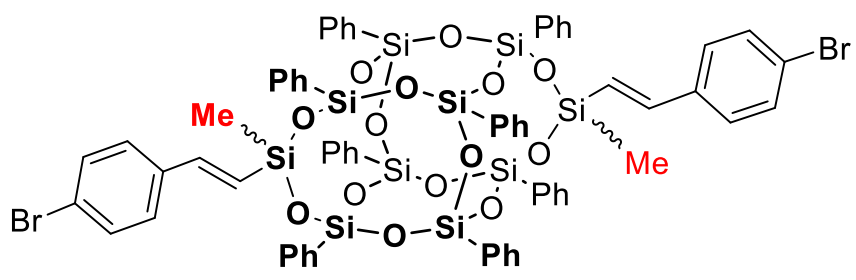

DDSQa White solid. Isolated Yield 82\%.

${ }^{1} \mathbf{H}$ NMR $\left(300 \mathrm{MHz}, \mathrm{CDCl}_{3}, \mathrm{ppm}\right): \delta=0.43\left(\mathrm{~s}, 6 \mathrm{H},-\mathrm{Si}_{-} \mathrm{CH}_{3}\right), 6.39$ (d, 2H, $\left.J=19.3 \mathrm{~Hz},=\mathrm{CH}-\mathrm{Si}\right), 6.94-7.46(\mathrm{~m}, 42 \mathrm{H}$, $\left.\mathrm{C}_{6} \mathrm{H}_{5^{-}},=\mathrm{CH}-\mathrm{C}_{6} \mathrm{H}_{4}-\mathrm{Br}\right), 7.57\left(\mathrm{~d}, 8 \mathrm{H}, J=7.2 \mathrm{~Hz}, \mathrm{C}_{6} \mathrm{H}_{4}-\mathrm{Br}\right)$.

${ }^{13} \mathrm{C}$ NMR (101 MHz, $\left.\mathrm{CDCl}_{3}, \mathrm{ppm}\right): \delta=-0.66\left(-\mathrm{Si}_{-} \mathrm{CH}_{3}\right), 122.45$ (ipso-C at $\mathrm{Br}$ of C6H4-Br), $125.14(=\mathrm{CH}-\mathrm{Si})$, 127.74-128.41, 130.52-131.98, 134.09-134.23, 136.61 (ipso-C of $\mathrm{C}_{6} \mathrm{H}_{4}-\mathrm{Br}$ ), 145.40 (-CH=Ar).

${ }^{29} \mathrm{Si}$ NMR (79 MHz, $\left.\mathrm{CDCl}_{3}, \mathrm{ppm}\right): \delta=-30.47$ (-Si-HC=CH-), -78.28, -79.31 (cis), -79.55 (trans), -79.77 (cis) ($\left.\mathrm{Si}-\mathrm{C}_{6} \mathrm{H}_{5}\right)$.

IR (ATR): $v=3071.77(\mathrm{~m}), 3049.73(\mathrm{~m}), 3026.31\left(\mathrm{~m} ; v_{\mathrm{s}}\left(\mathrm{C}-\mathrm{H}\right.\right.$ phenyl)), $2963.31\left(\mathrm{~m} ; v_{\mathrm{s}}(\mathrm{C}-\mathrm{H})\right), 1606.00(\mathrm{~m}$; $\left.v_{\mathrm{s}}(\mathrm{C}=\mathrm{C})\right), 1593.61(\mathrm{~m}), 1485.47(\mathrm{~m}), 1429.70(\mathrm{~m}), 1261.96(\mathrm{~m}), 1177.78(\mathrm{~s}), 1028.63\left(\mathrm{~s} ; v_{\mathrm{as}}(\mathrm{Si}-\mathrm{O}-\mathrm{Si}), 997.96 \mathrm{~cm}^{-}\right.$ 1 (s).

MALDI-ToF MS: Calcd. for $\mathrm{C}_{66} \mathrm{H}_{58} \mathrm{Br}_{2} \mathrm{Na}^{+} \mathrm{O}_{14} \mathrm{Si}_{10}: m / z$ 1534.9778 [M + $\left.\mathrm{Na}^{+}\right]$. Found: 1534.9791.

Elemental analysis: Found: $\mathrm{C}, 52.4 ; \mathrm{H}, 3$.43.Calc. for $\mathrm{C}_{66} \mathrm{H}_{58} \mathrm{Br}_{2} \mathrm{O}_{14} \mathrm{Si}_{10}$ : C, 52.3; H, 3.86\% Analytical data correspond with the literature. ${ }^{2}$
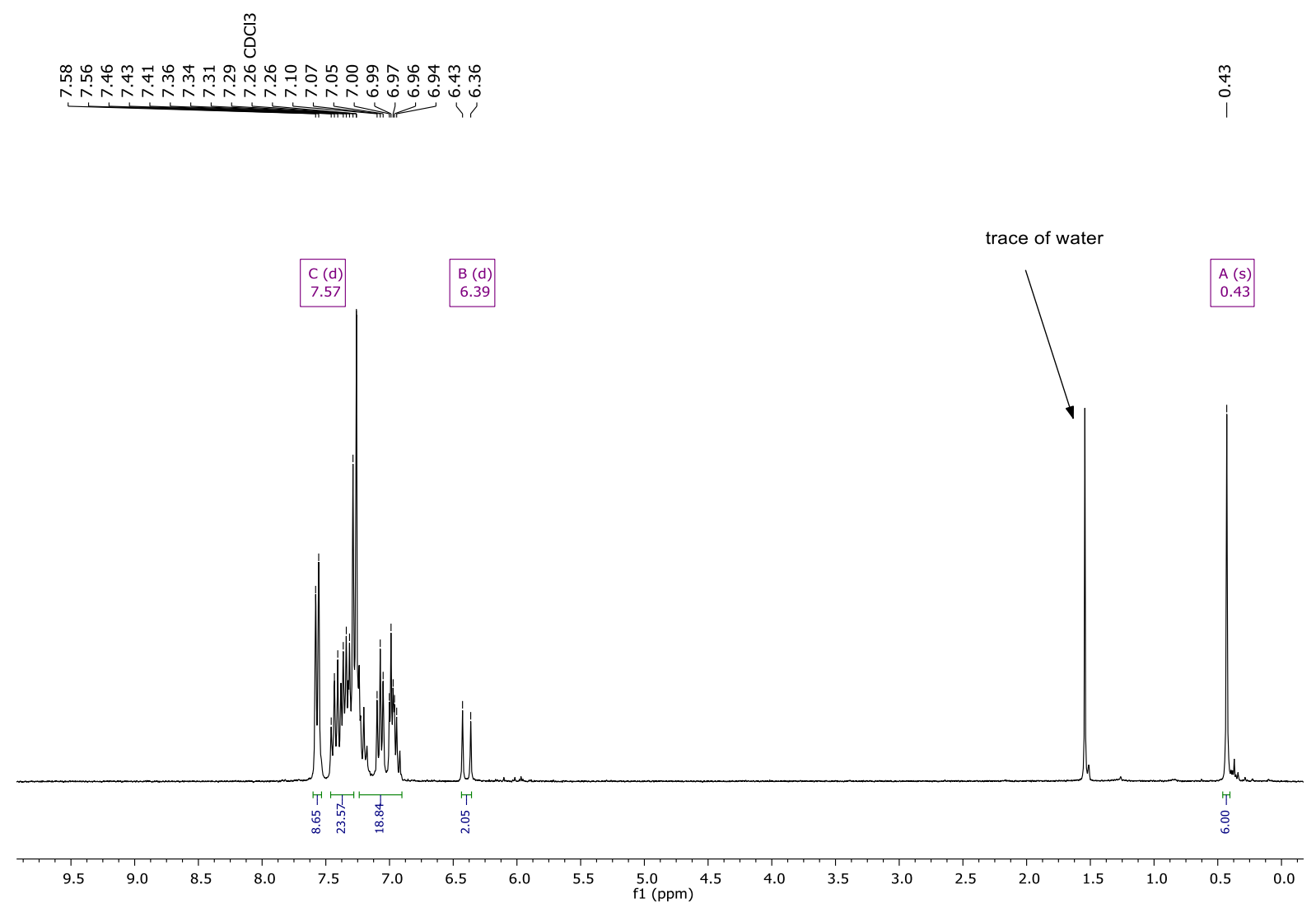

Figure S1. ${ }^{1} \mathrm{H}$ NMR spectra of DDSQa $\left(300 \mathrm{MHz}, \mathrm{CDCl}_{3}\right)$. 


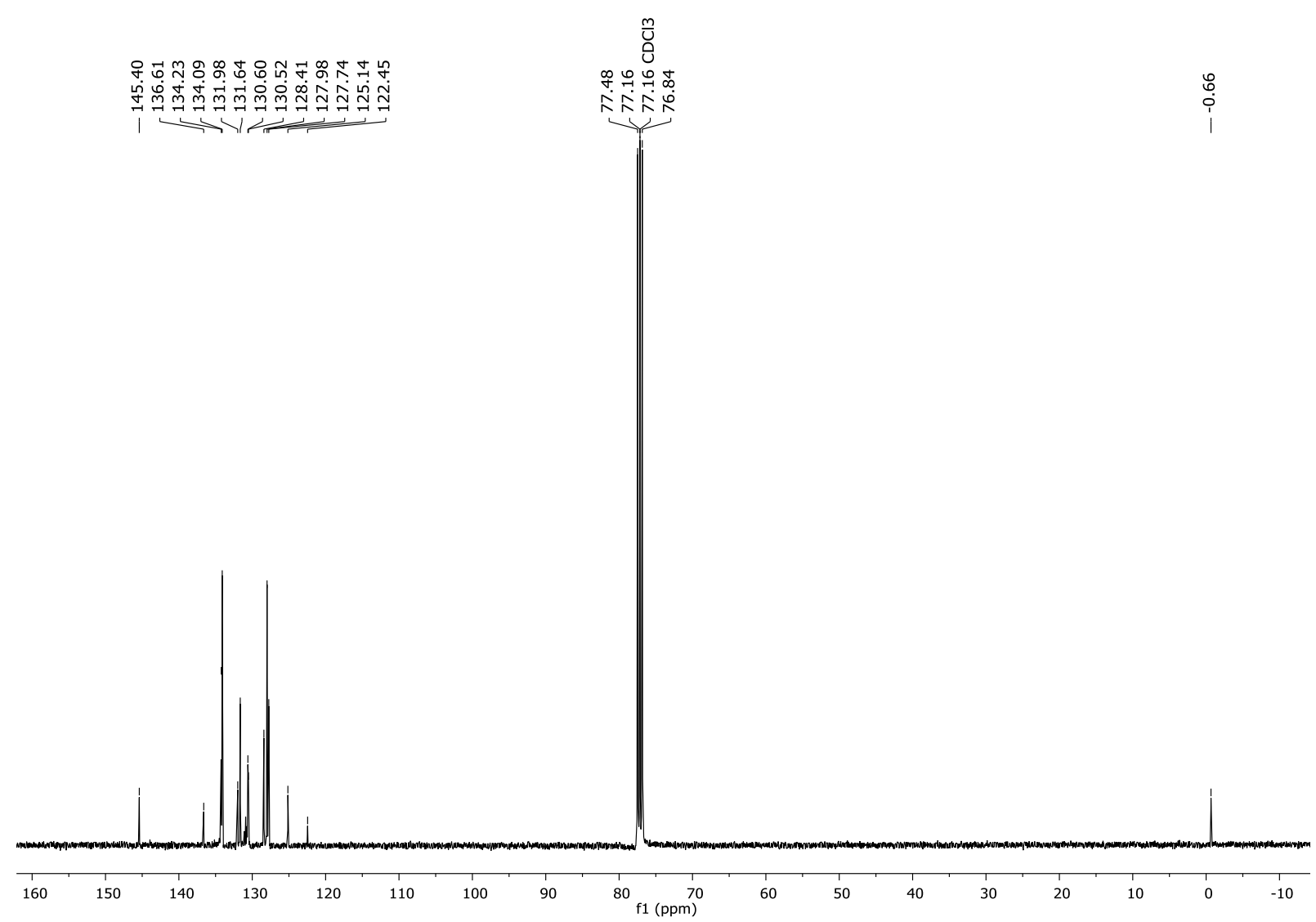

Figure S2. ${ }^{13} \mathrm{C}$ NMR spectra of DDSQa $\left(101 \mathrm{MHz}, \mathrm{CDCl}_{3}\right)$.
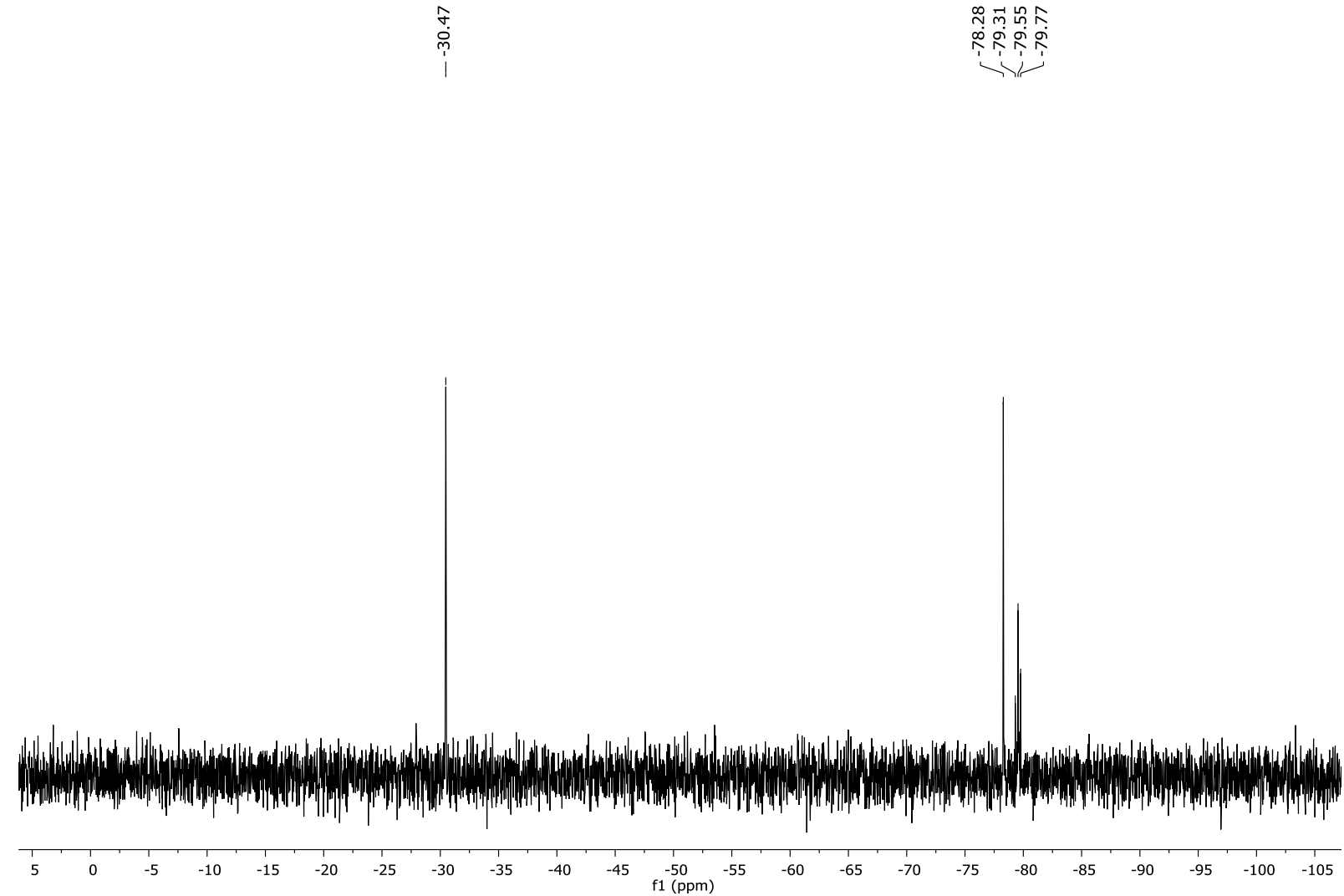

Figure S3. ${ }^{29} \mathrm{Si}$ NMR spectra of DDSQa $\left(79 \mathrm{MHz}, \mathrm{CDCl}_{3}\right)$. 


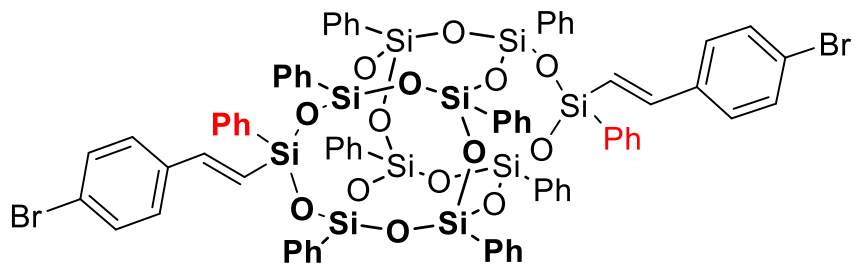

DDSQb White solid. Isolated Yield 90\%.

${ }^{1} \mathbf{H}$ NMR $\left(300 \mathrm{MHz}, \mathrm{CDCl}_{3}, \mathrm{ppm}\right): \delta=6.51(\mathrm{~d}, 2 \mathrm{H}, J=19.2 \mathrm{~Hz}$, $=\mathrm{CH}-\mathrm{Si}), 6.97-7.70\left(\mathrm{~m}, 60 \mathrm{H}, \mathrm{C}_{6} H_{5}-\right.$ and $\mathrm{C}_{6} H_{4}-\mathrm{Br}$, $\left.=\mathrm{CH}-\mathrm{C}_{6} \mathrm{H}_{4}-\mathrm{Br}\right)$.

${ }^{13} \mathbf{C}$ NMR (101 MHz, $\mathrm{CDCl}_{3}, \mathrm{ppm}$ ): $\delta=122.62$ (ipso-C at $\mathrm{Br}$ of $\mathrm{C}_{6} \mathrm{H}_{4}-\mathrm{Br}$ ), 123.58 (=CH-Si), 127.67-128.52, 130.42-131.71, 134.16-134.24, 136.49 (ipso-C of $\left.\mathrm{C}_{6} \mathrm{H}_{4}-\mathrm{Br}\right), 146.81(\mathrm{CH}=\mathrm{Ar})$.

${ }^{29} \mathrm{Si}$ NMR $\left(79 \mathrm{MHz}, \mathrm{CDCl}_{3}, \mathrm{ppm}\right): \delta=-44.83(-\mathrm{Si}-\mathrm{HC}=\mathrm{CH}-),-77.93,-79.41$ (trans) $\left(-\mathrm{Si}_{-} \mathrm{C}_{6} \mathrm{H}_{5}\right)$.

IR (ATR) $v=3071.26(\mathrm{~m}), 3045.28(\mathrm{~m}), 3005.40\left(\mathrm{~m} ; v_{\mathrm{s}}\left(\mathrm{C}-\mathrm{H}\right.\right.$ phenyl)), $1593.49\left(\mathrm{w} ; v_{\mathrm{s}}(\mathrm{C}=\mathrm{C})\right), 1485.34(\mathrm{~m})$, $1429.22(\mathrm{~m}), 1264.56(\mathrm{~m}), 1064.69$ (s), 1027.21 (s; $v_{a s}(\mathrm{Si}-\mathrm{O}-\mathrm{Si}), 996.85 \mathrm{~cm}^{-1}$ (s).

MALDI-ToF MS: Calcd. for $\mathrm{C}_{76} \mathrm{H}_{62} \mathrm{Br}_{2} \mathrm{Na}^{+} \mathrm{O}_{14} \mathrm{Si}_{10}: m / z 1659.0091$ [M $\left.+\mathrm{Na}^{+}\right]$. Found: 1659.0107.

Elemental analysis: Found: $\mathrm{C}, 55.53 ; \mathrm{H}, 3.66$. Calc for $\mathrm{C}_{76} \mathrm{H}_{62} \mathrm{Br}_{2} \mathrm{O}_{14} \mathrm{Si}_{10}$ : $\mathrm{C}, 55.66 ; \mathrm{H}, 3.81 \%$

Crystal Data for $\mathrm{C}_{76} \mathrm{H}_{62} \mathrm{Br}_{2} \mathrm{O}_{14} \mathrm{Si}_{10}(M=1639.97 \mathrm{~g} / \mathrm{mol})$ : monoclinic, space group $\mathrm{P} 2_{1} / \mathrm{c}$ (no. 14), $a=13.8835(4) \AA$, $b=13.9833(2) \AA, c=19.2735(5) \AA, \beta=92.132(2)^{\circ}, V=3739.11(15) \AA^{3}, Z=2, T=100.01(10) \mathrm{K}, \mu(\mathrm{MoK \alpha})=$ $1.309 \mathrm{~mm}^{-1}$, Dcalc $=1.457 \mathrm{~g} / \mathrm{cm}^{3}, 57797$ reflections measured $\left(5.826^{\circ} \leq 2 \Theta \leq 57.168^{\circ}\right), 8758$ unique $\left(R_{\text {int }}=0.0270\right.$, $\mathrm{R}_{\text {sigma }}=0.0211$ ) which were used in all calculations. The final $R_{1}$ was $0.0613\left(\mathrm{I}>2 \sigma(\mathrm{I})\right.$ ) and $w R_{2}$ was 0.1796 (all data).

CCDC 2048724 contains the supplementary crystallographic data for this paper. These data can be obtained free of charge from The Cambridge Crystallographic Data Centre via www.ccdc.cam.ac.uk/data_request/cif. Analytical data correspond with the literature. ${ }^{2}$

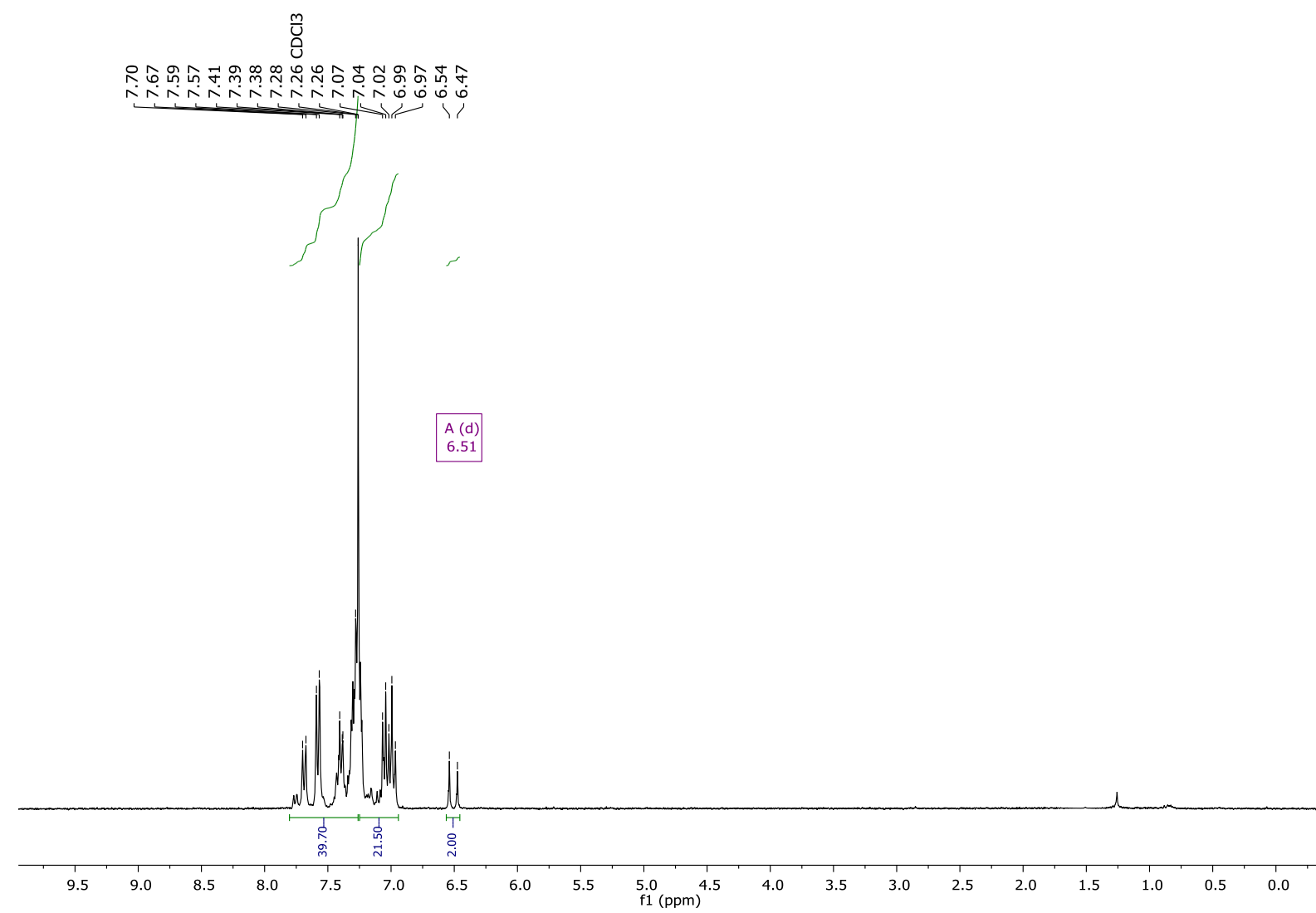

Figure S4. ${ }^{1} \mathrm{H}$ NMR spectra of DDSQb $\left(300 \mathrm{MHz}, \mathrm{CDCl}_{3}\right)$. 


$$
\pm
$$




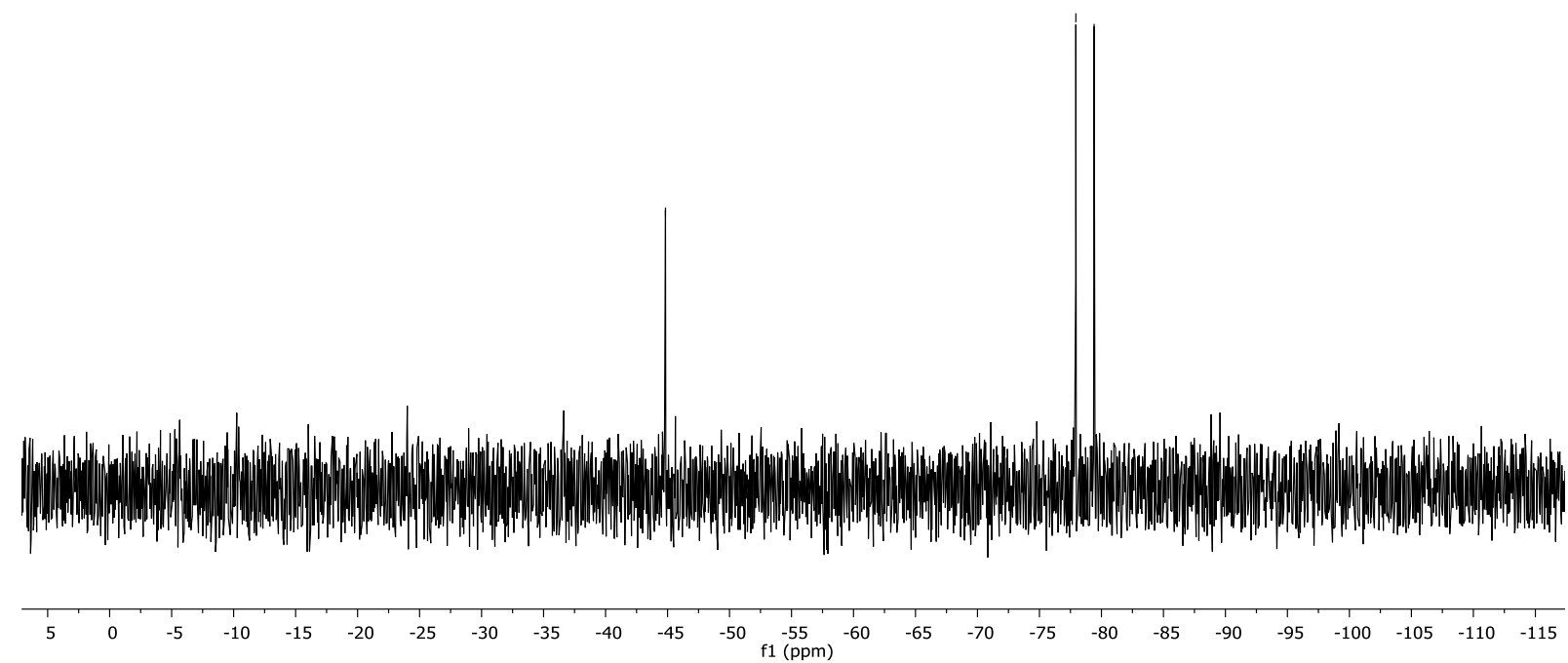

Figure S7. ${ }^{29} \mathrm{Si} \mathrm{NMR} \mathrm{spectra} \mathrm{of} \mathrm{DDSQb}\left(79 \mathrm{MHz}, \mathrm{CDCl}_{3}\right)$. 


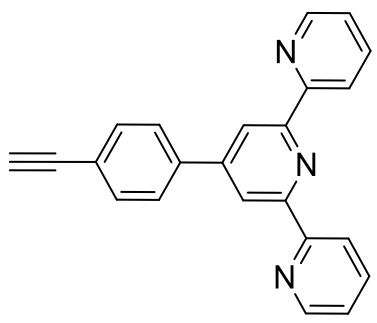

4'-(4-Ethylnylphenyl)-[2,2':6,2'’]terpyridine, T. Pale yellow solid. Isolated Yield 70\%.

${ }^{1} \mathrm{H}$ NMR (300 MHz, $\left.\mathrm{CDCl}_{3}, \mathrm{ppm}\right): \delta=3.19$ (s, 1H, HC $\left.\equiv\right)$, 7.34-7.39 (m, 2H), 7.64 (d, 2H, J=8.3 Hz), 7.87-7.92 $(\mathrm{m}, 4 \mathrm{H}), 8.68(\mathrm{~d}, 2 \mathrm{H}, J=7.9 \mathrm{~Hz}), 8.73(\mathrm{~s}, 4 \mathrm{H})$.

${ }^{13} \mathrm{C} \mathrm{NMR}\left(101 \mathrm{MHz}, \mathrm{CDCl}_{3}, \mathrm{ppm}\right): \delta=78.67(\mathrm{HC} \equiv), 83.40(\equiv \mathrm{C}-), 118.85,121.51,122.94,124.05,127.38,132.83$, $137.03,138.97,149.29,149.43,156.22,156.23$.

Analytical data correspond with the literature. ${ }^{1}$

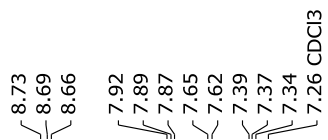

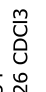
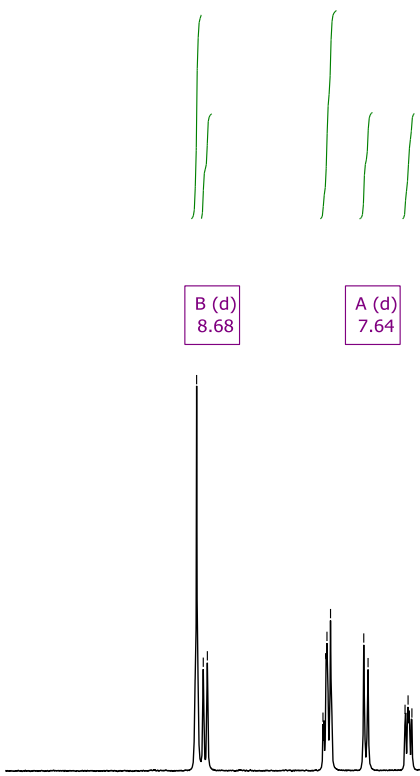

trace of water

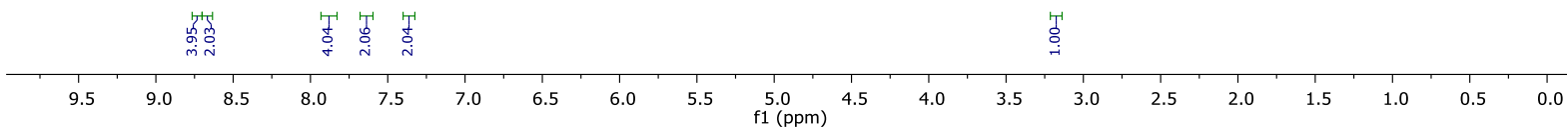

Figure S8. ${ }^{1} \mathrm{H}$ NMR spectra of $\mathrm{T}\left(300 \mathrm{MHz}, \mathrm{CDCl}_{3}\right)$. 


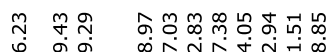

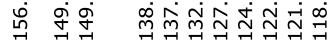

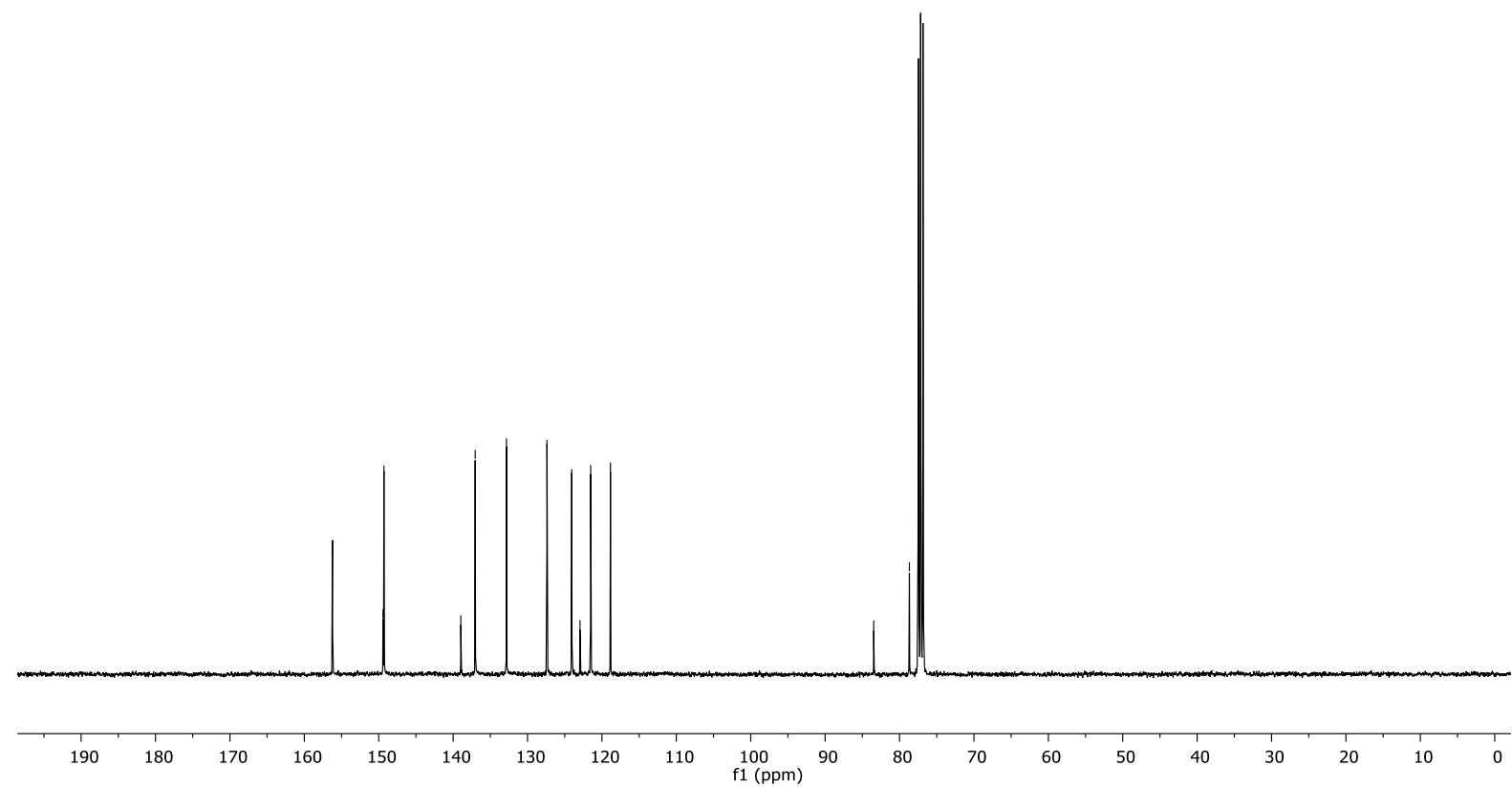

Figure S9. ${ }^{13} \mathrm{C}$ NMR spectra of $\mathrm{T}\left(101 \mathrm{MHz}, \mathrm{CDCl}_{3}\right)$. 


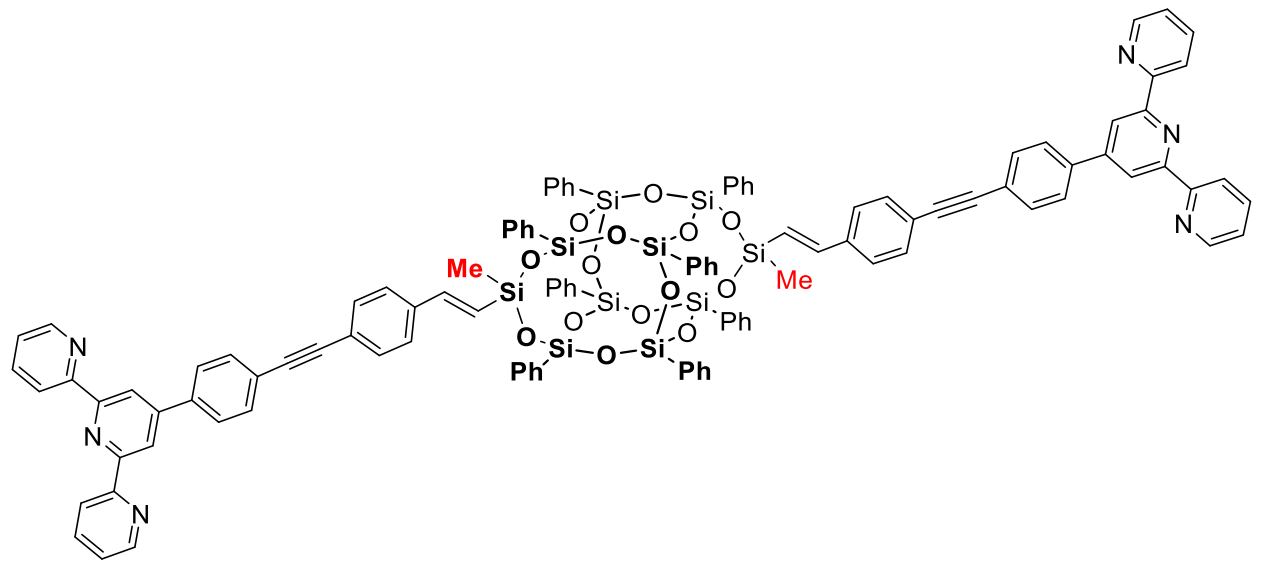

DDSQ_Ta Brown solid. Isolated Yield 68\%.

${ }^{1} \mathbf{H}$ NMR $\left(400 \mathrm{MHz}, \mathrm{CDCl}_{3}, \mathrm{ppm}\right): \delta=0.44\left(\mathrm{~s}, 6 \mathrm{H},-\mathrm{Si}_{-} \mathrm{CH}_{3}\right), 6.44-6.49$ (m, 2H, =CH-Si), 6.98-8.72 (m, 78H, $\mathrm{C}_{6} \mathrm{H}_{5}$ - and $\left.-\mathrm{C}_{6} \mathrm{H}_{4-},=\mathrm{CH}-\mathrm{C}_{6} \mathrm{H}_{4-}, \mathrm{Tpy}\right)$.

MAS ${ }^{13}$ C NMR (125 MHz, ppm): $\delta=-0.56\left(-\mathrm{Si}_{-} \mathrm{CH}_{3}\right), 123.2-133.39,147.52,155.42(\mathrm{Ph}, \mathrm{Tpy})$.

MAS ${ }^{29}$ Si NMR (99.3 MHz, ppm): $\delta=-35.99(-\mathrm{Si}-\mathrm{HC}=\mathrm{CH}-),-84.94\left(-\mathrm{Si}_{-} \mathrm{C}_{6} \mathrm{H}_{5}\right)$.

IR (ATR): $v=3070.45(\mathrm{~m}), 3048.67\left(\mathrm{~m}, v_{\mathrm{s}}\left(\mathrm{C}-\mathrm{H}\right.\right.$ phenyl)), $2962.61\left(\mathrm{~m}, v_{\mathrm{s}}(\mathrm{C}-\mathrm{H})\right), 1601.98\left(\mathrm{~m} ; v_{\mathrm{s}}(\mathrm{C}=\mathrm{C})\right), 1583.32$ $(\mathrm{m}), 1565.60\left(\mathrm{~m} ; v_{\mathrm{s}}(\mathrm{C}=\mathrm{N})\right), 1516.44(\mathrm{~m}), 1466.56(\mathrm{~m}), 1429.55(\mathrm{~m}), 1387.79(\mathrm{~m}), 1262.99(\mathrm{~m}), 1074.11(\mathrm{~s})$, 1027.73 (s; vas $(\mathrm{Si}-\mathrm{O}-\mathrm{Si}), 996.98 \mathrm{~cm}^{-1}(\mathrm{~s})$.

MALDI-ToF MS: Calcd. for $\mathrm{C}_{112} \mathrm{H}_{86} \mathrm{H}^{+} \mathrm{N}_{6} \mathrm{O}_{14} \mathrm{Si}_{10}: \mathrm{m} / z$ 2019.3967 [M + $\left.\mathrm{H}^{+}\right]$. Found: 2019.4044.

Elemental analysis: Found: $\mathrm{C}, 61.79 ; \mathrm{H}, 4.06 ; \mathrm{N}, 3.98$. Calc for $\mathrm{C}_{112} \mathrm{H}_{86} \mathrm{~N}_{6} \mathrm{O}_{14} \mathrm{Si}_{10}$ : C, 66.57; H, 4.29; $\mathrm{N}, 4.16 \%$

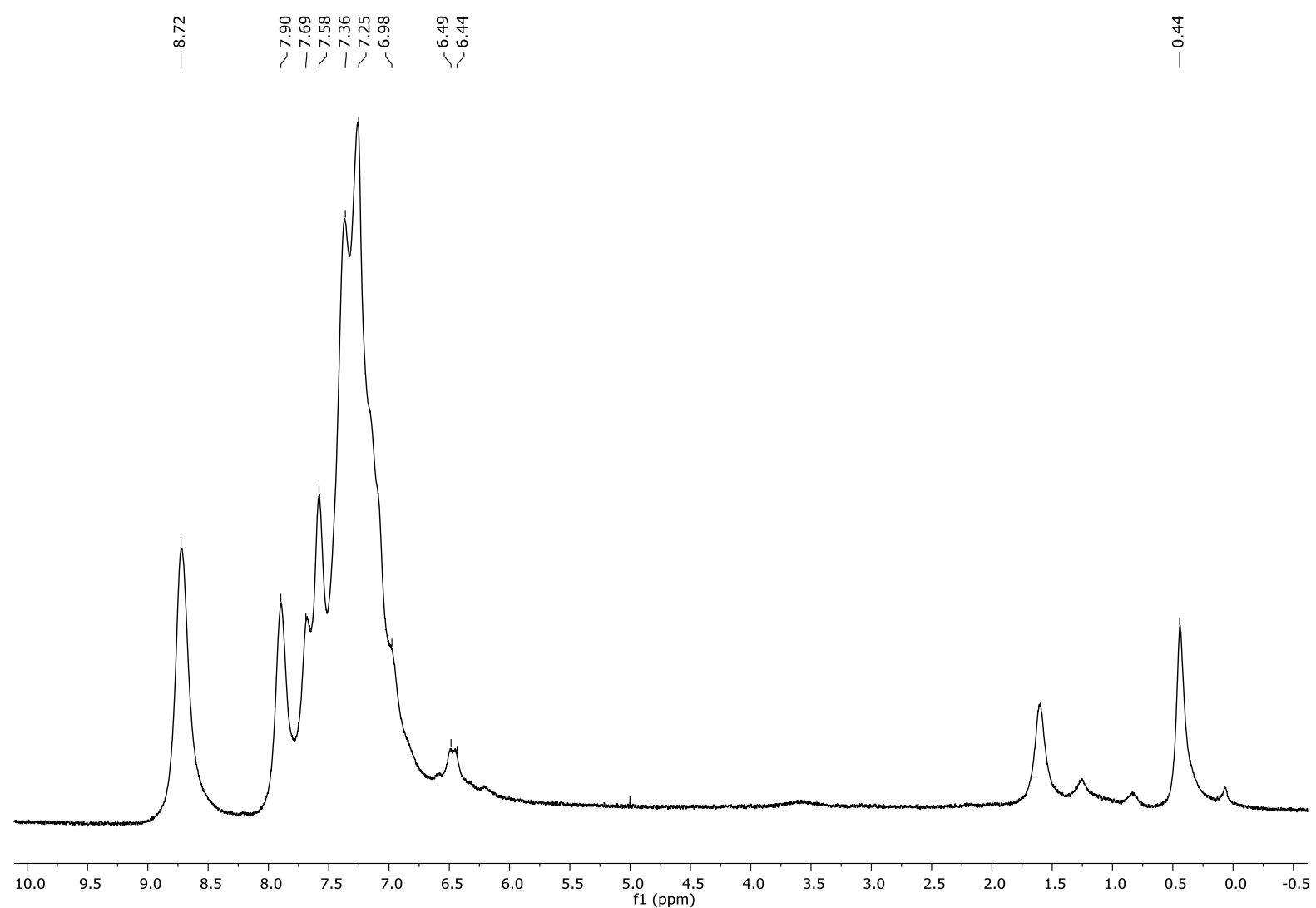

Figure S10. ${ }^{1} \mathrm{H}$ NMR spectra of DDSQ_Ta $\left(400 \mathrm{MHz}, \mathrm{CDCl}_{3}\right)$ 


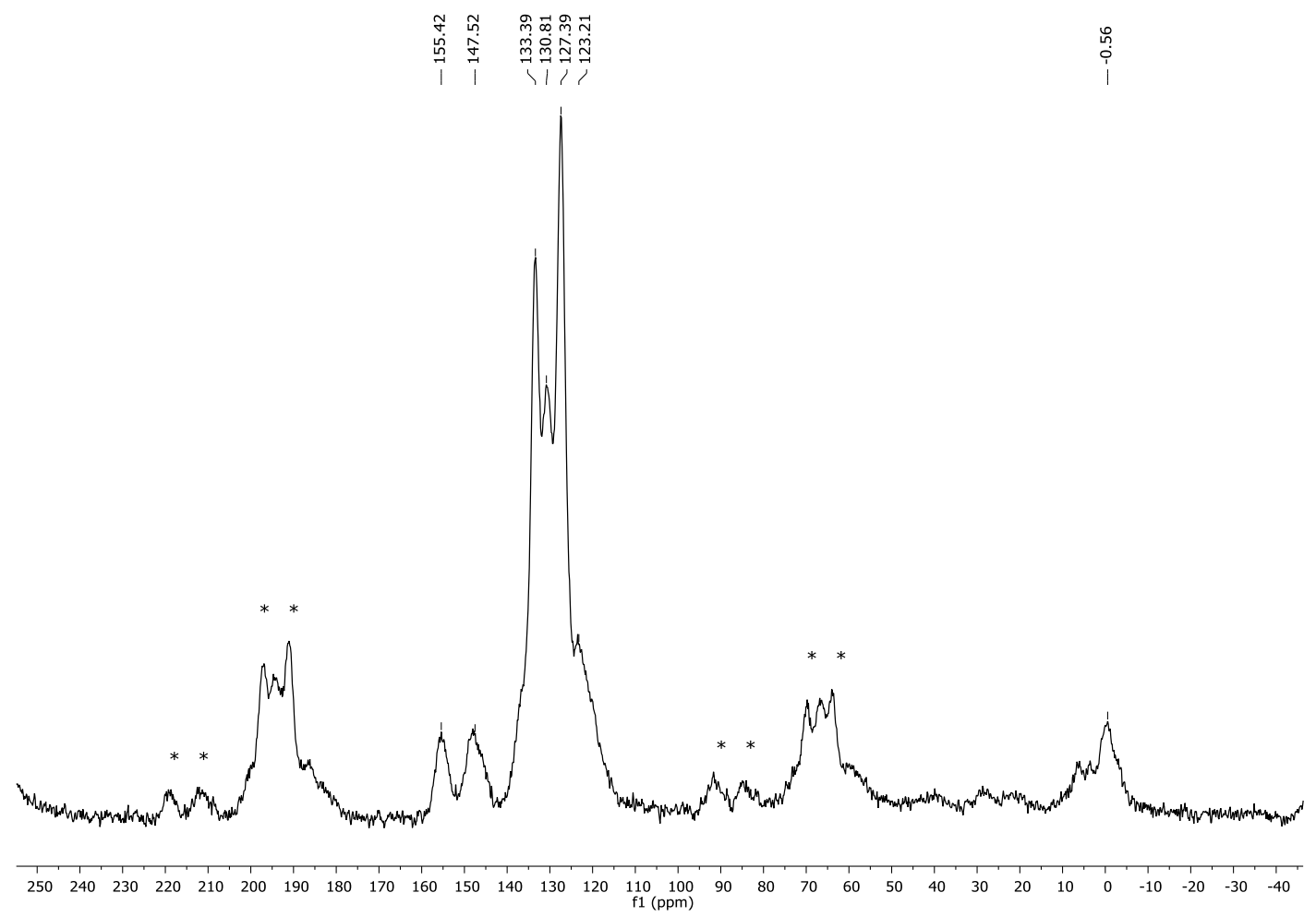

Figure S11. CP-MAS ${ }^{13} \mathrm{C}$ NMR spectra of DDSQ_Ta (125 MHz, 8kHz). Asterisks denote spinning sidebands.

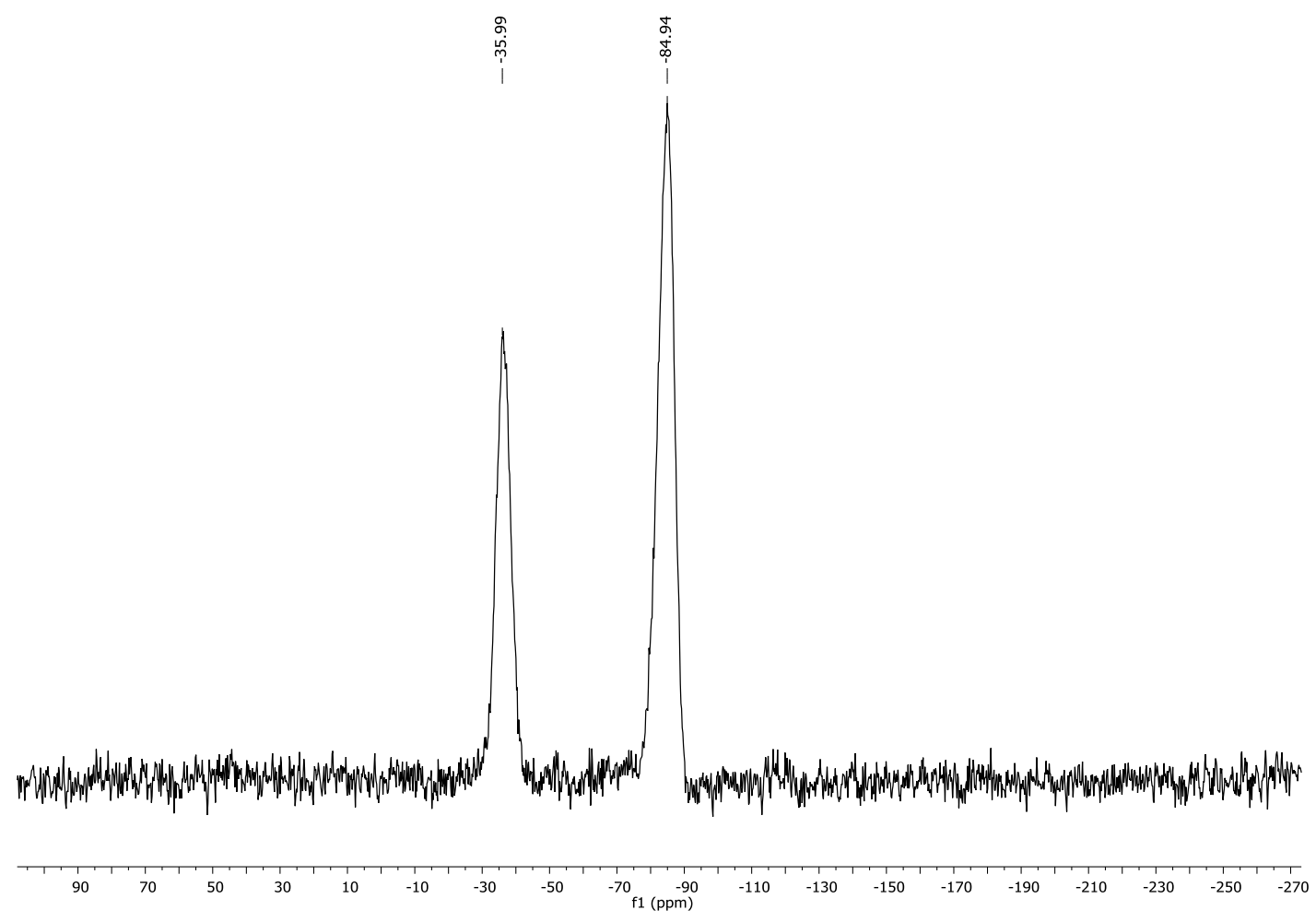

Figure S12. CP-MAS ${ }^{29} \mathrm{Si}$ NMR spectra of DDSQ_Ta $(99.3 \mathrm{MHz}, 8 \mathrm{kHz})$. 


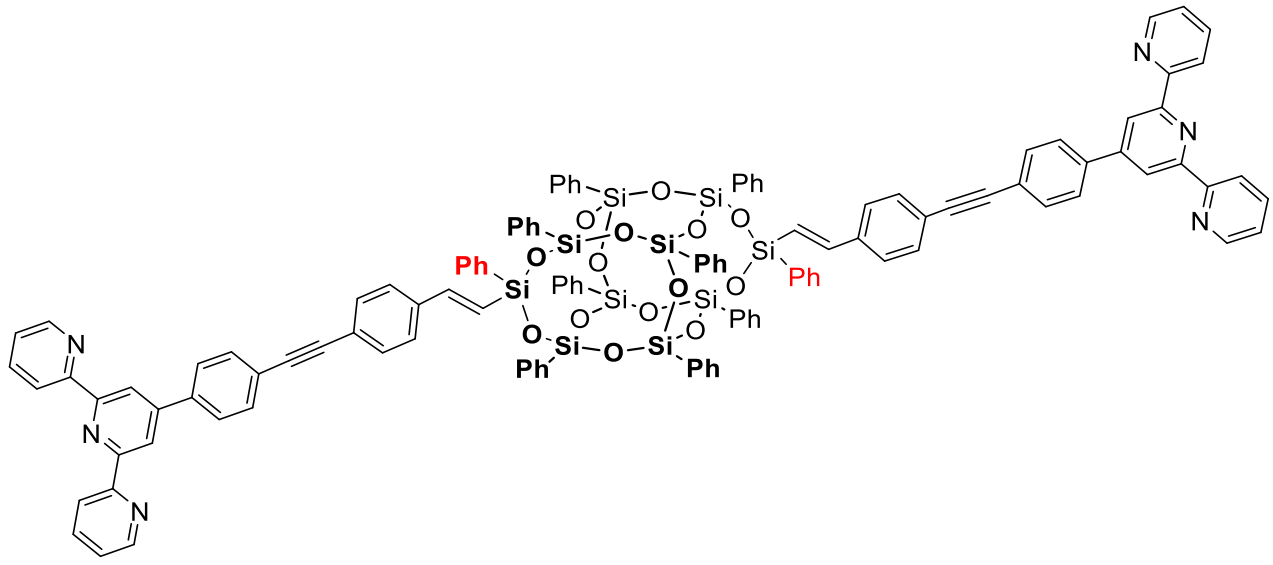

DDSQ_Tb Brown solid. Isolated Yield 72\%.

${ }^{1} \mathbf{H}$ NMR $\left(400 \mathrm{MHz}, \mathrm{CDCl}_{3}, \mathrm{ppm}\right): \delta=6.57-6.62(\mathrm{~m}, 2 \mathrm{H},=\mathrm{CH}-\mathrm{Si}), 7.12-8.74\left(\mathrm{~m}, 88 \mathrm{H}, \mathrm{C}_{6} H_{5^{-}}\right.$and $\mathrm{C}_{6} \mathrm{H}_{4-},{ }=\mathrm{CH}-\mathrm{C}_{6} \mathrm{H}_{4-}$, Tpy).

MAS ${ }^{13}$ C NMR (125 MHz, ppm): $\delta=112.94-131.13,145.28,152.91$ (Ph, Tpy).

MAS ${ }^{29} \mathrm{Si}$ NMR (99.3 MHz, ppm): $\delta=-50.71(-\mathrm{Si}-\mathrm{HC}=\mathrm{CH}-),-84.21\left(-\mathrm{Si}_{-} \mathrm{C}_{6} \mathrm{H}_{5}\right)$.

IR (ATR): $v=3070.21(\mathrm{~m}), 3048.66\left(\mathrm{~m}, v_{\mathrm{s}}\left(\mathrm{C}-\mathrm{H}\right.\right.$ phenyl)), $1600.00\left(\mathrm{~m} ; v_{\mathrm{s}}(\mathrm{C}=\mathrm{C})\right), 1583.47(\mathrm{~m}), 1564.94\left(\mathrm{~m} ; v_{\mathrm{s}}\right.$ $(\mathrm{C}=\mathrm{N})), 1516.30(\mathrm{~m}), 1465.80(\mathrm{~m}), 1429.20(\mathrm{~m}), 1387.49(\mathrm{~m}), 1262.99(\mathrm{~m}), 1072.80(\mathrm{~s}), 1027.17$ (s; $v_{\text {as }}(\mathrm{Si}-\mathrm{O}-\mathrm{Si})$, $996.70 \mathrm{~cm}^{-1}(\mathrm{~s})$.

MALDI-ToF MS: Calcd. for $\mathrm{C}_{122} \mathrm{H}_{90} \mathrm{H}^{+} \mathrm{N}_{6} \mathrm{O}_{14} \mathrm{Si}_{10}: m / z 2143.4280\left[\mathrm{M}+\mathrm{H}^{+}\right]$. Found: 2143.4058 .

Elemental analysis: Found: $\mathrm{C}, 67.07 ; \mathrm{H}, 4.04 ; \mathrm{N}, 4.08$. Calc for $\mathrm{C}_{122} \mathrm{H}_{90} \mathrm{~N}_{6} \mathrm{O}_{14} \mathrm{Si}_{10}$ : C, 68,32; H, 4.23; N, 3.92\%

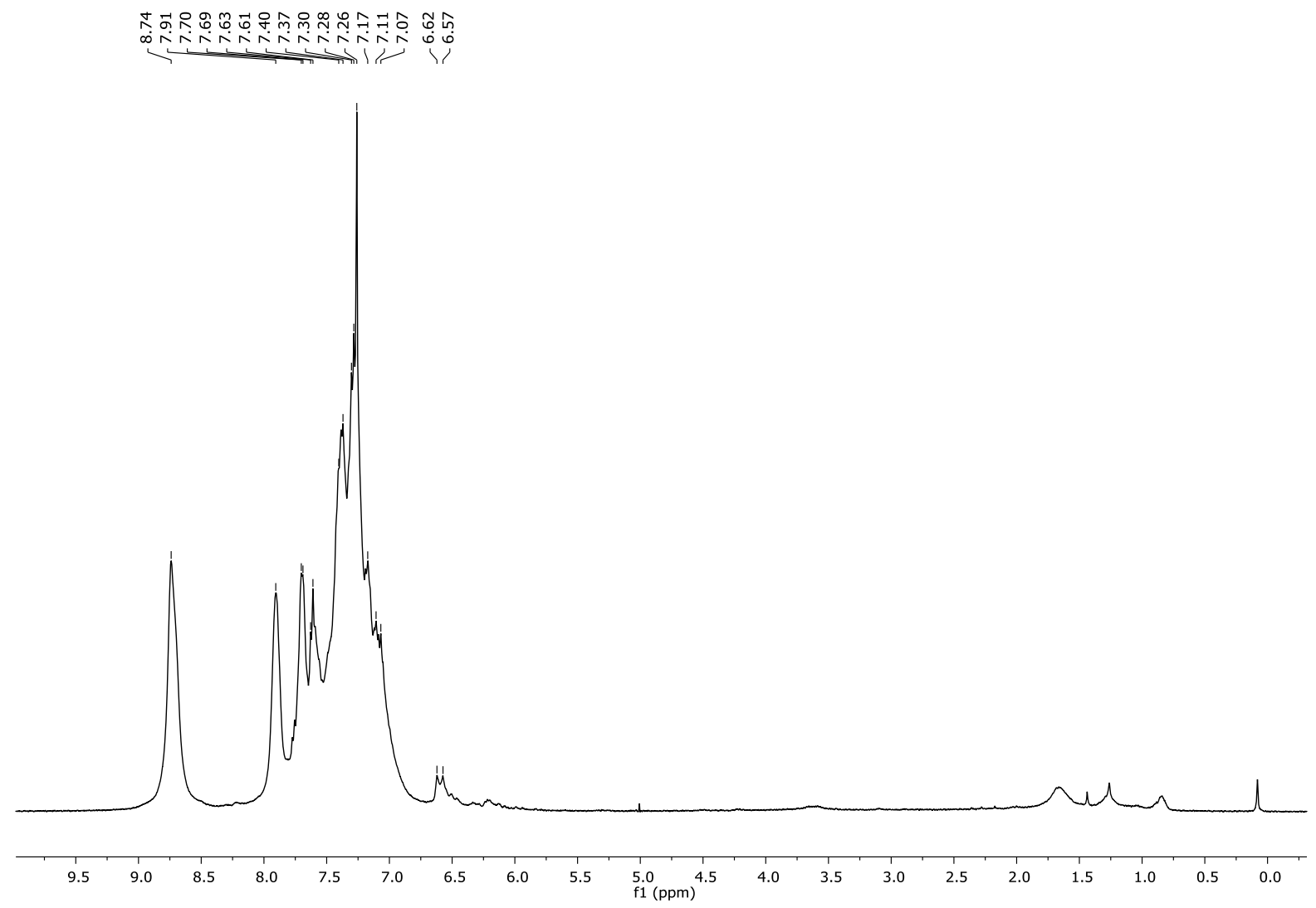

Figure S13. ${ }^{1} \mathrm{H}$ NMR spectra of DDSQ_Tb $\left(400 \mathrm{MHz}, \mathrm{CDCl}_{3}\right)$ 


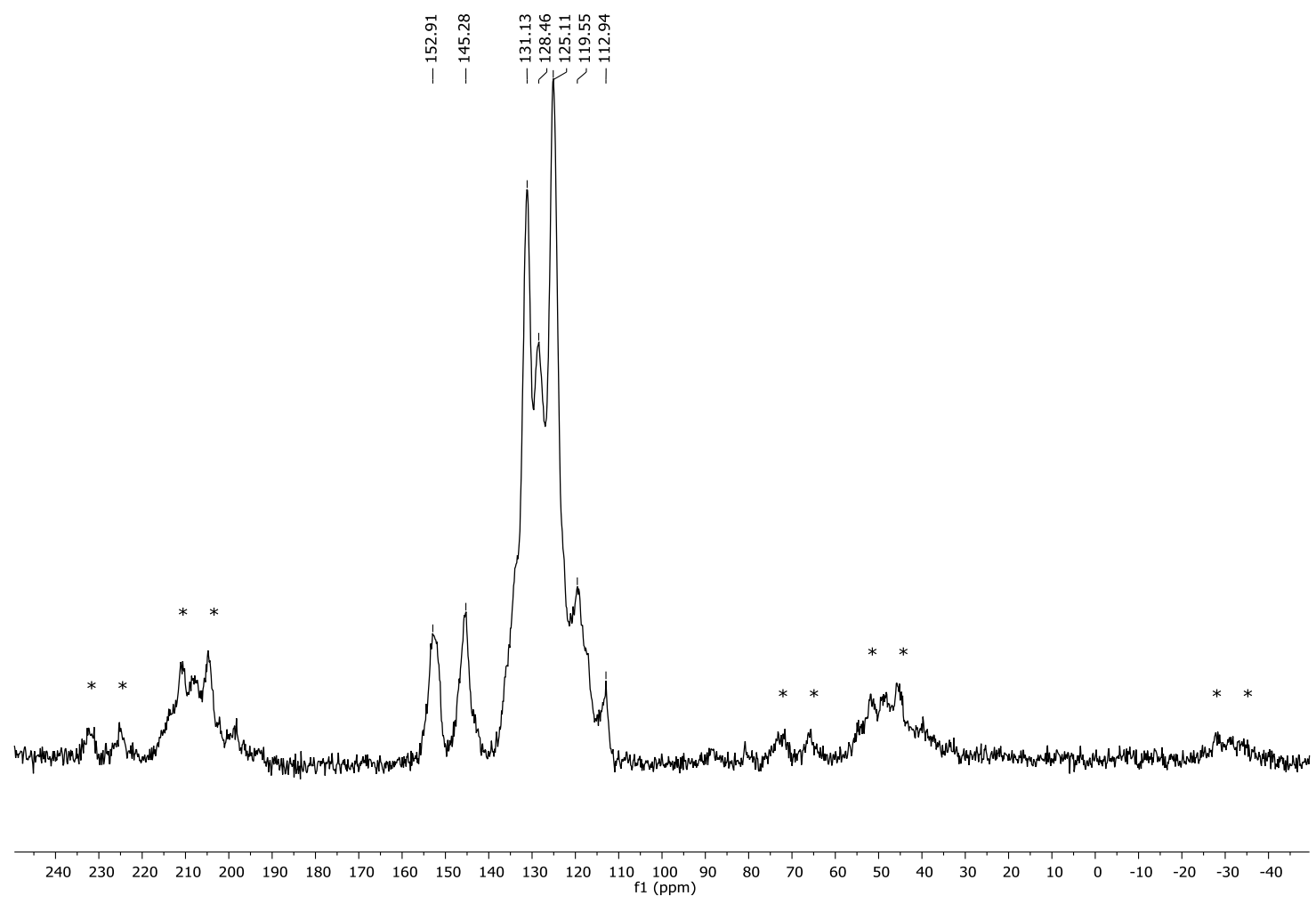

Figure S14. CP-MAS ${ }^{13} \mathrm{C}$ NMR spectra of DDSQ_Tb $(125 \mathrm{MHz}, 8 \mathrm{kHz})$. Asterisks denote spinning sidebands.

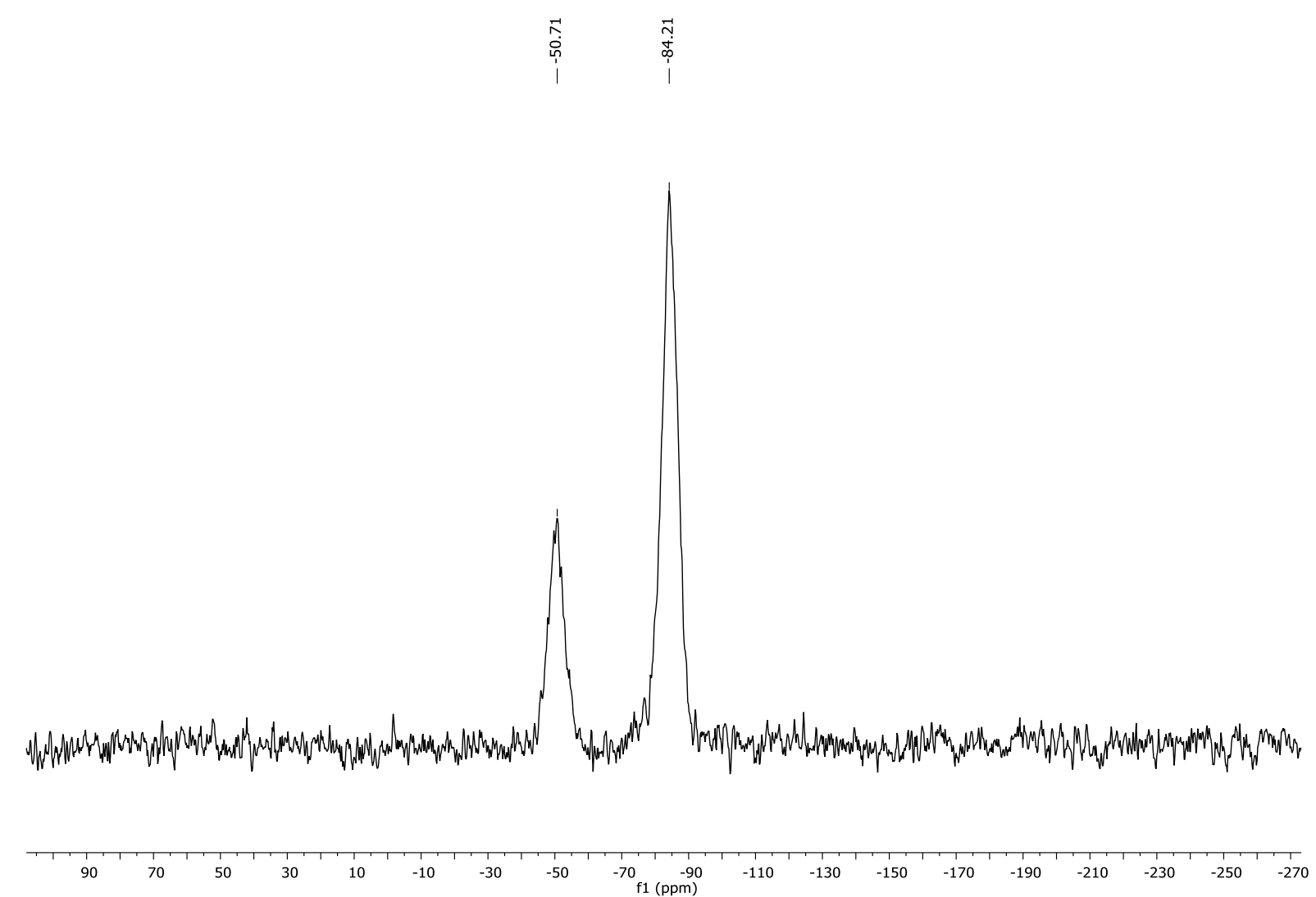

Figure S15. CP-MAS ${ }^{29} \mathrm{Si}$ NMR spectra of DDSQ_Tb $(99.3 \mathrm{MHz}, 8 \mathrm{kHz})$. 


\subsection{Thermal analysis of reagents DDSQa-b and DDSQ_Ta-b}

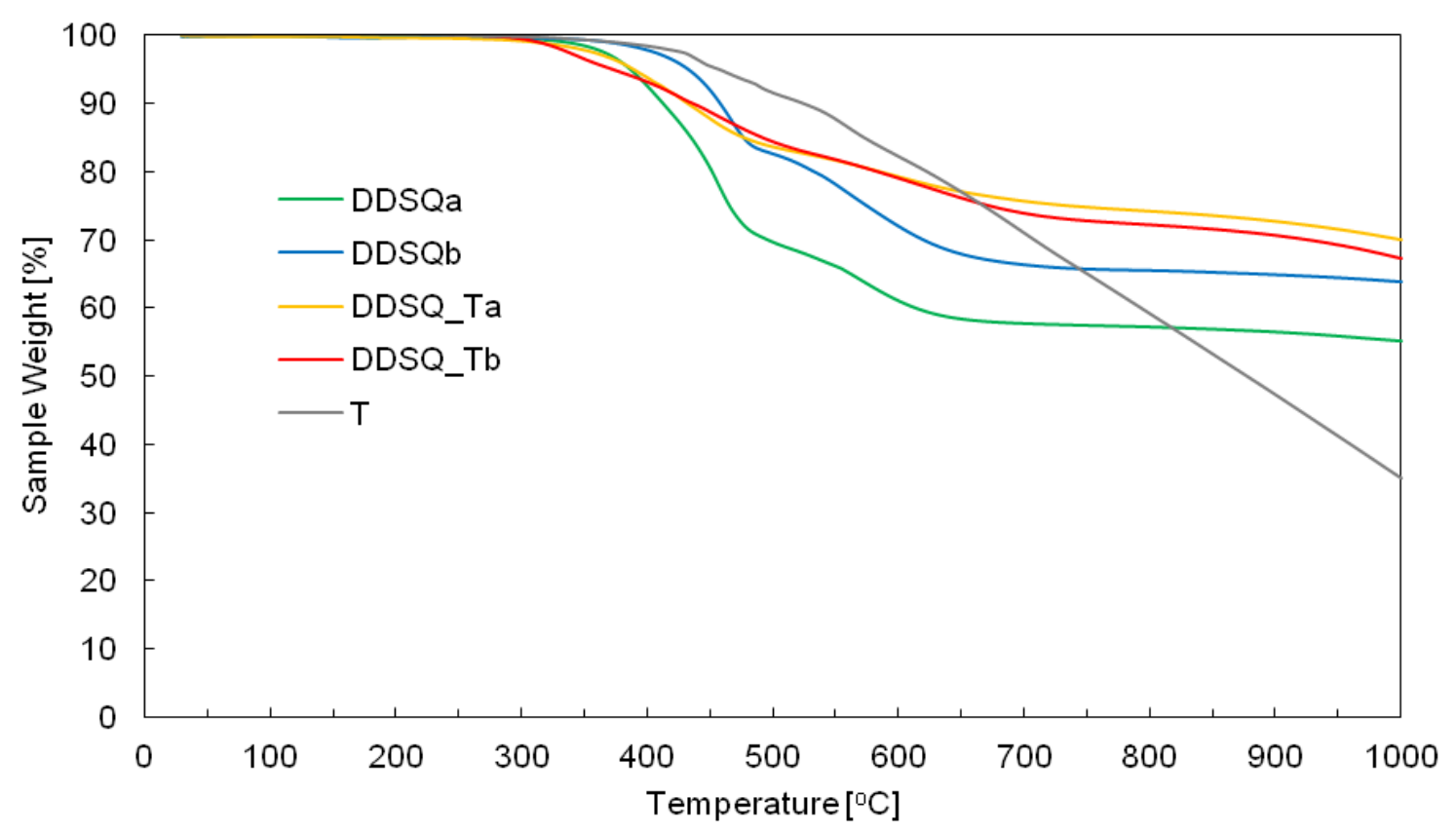

Figure S16. TGA analysis of reagents performed in nitrogen atmosphere.

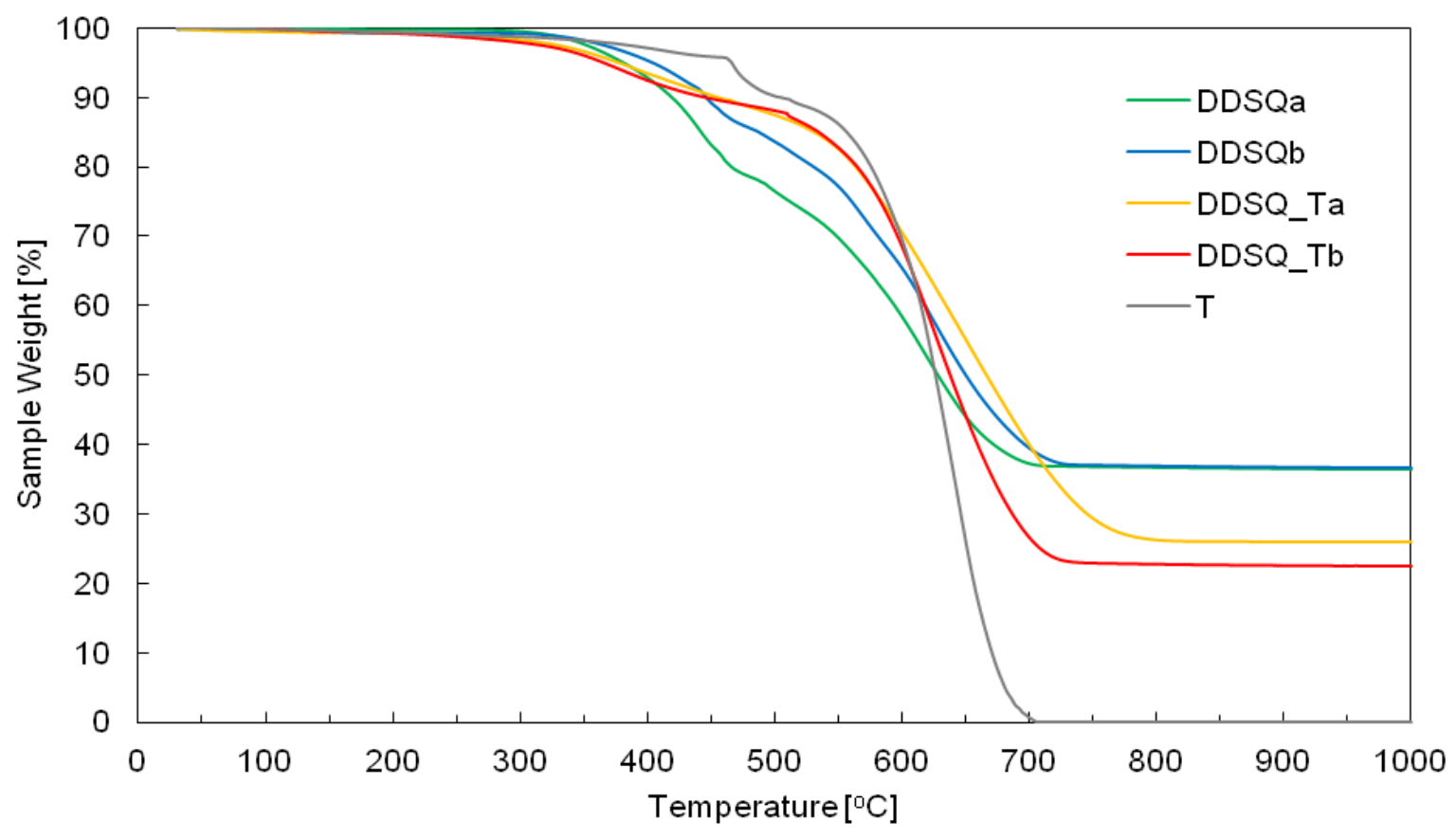

Figure S17. TGA analysis of reagents performed in air atmosphere. 


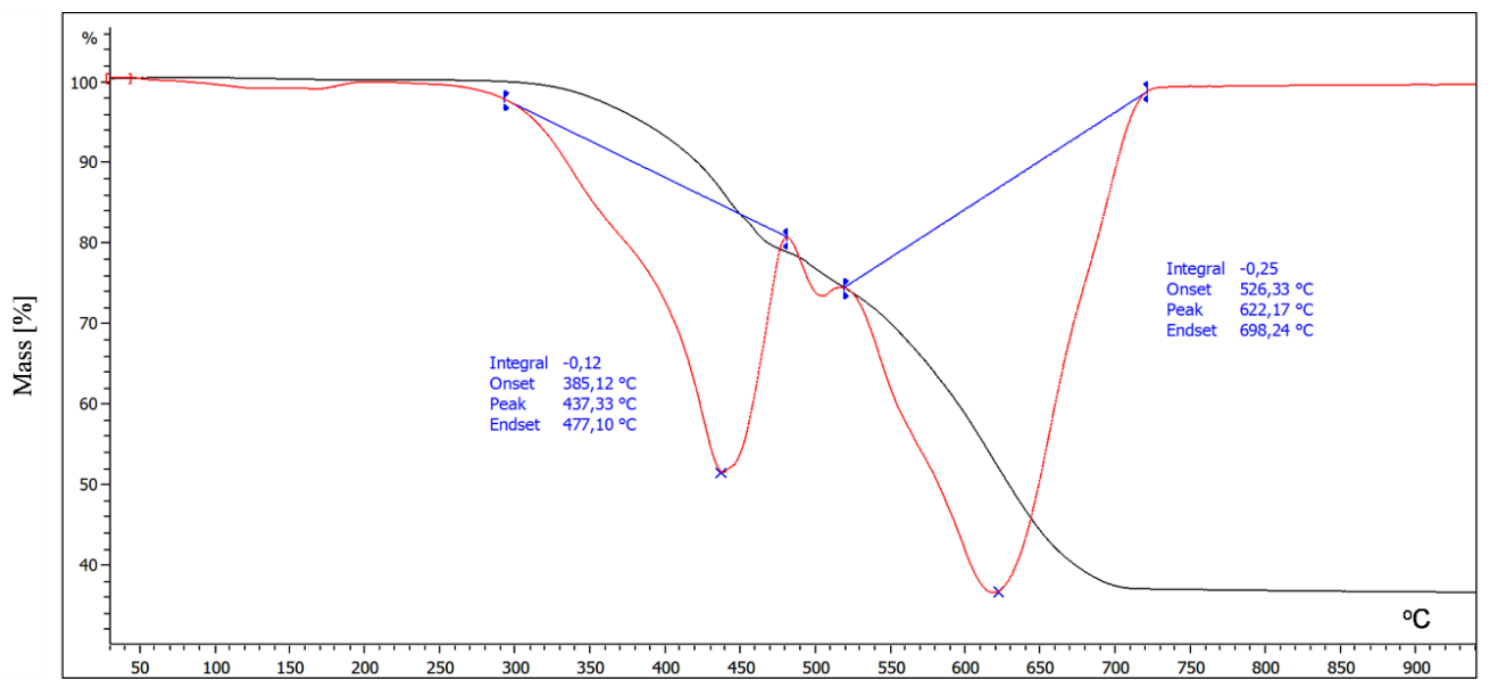

Figure S18. DTG curves of DDSQa in air atmosphere.

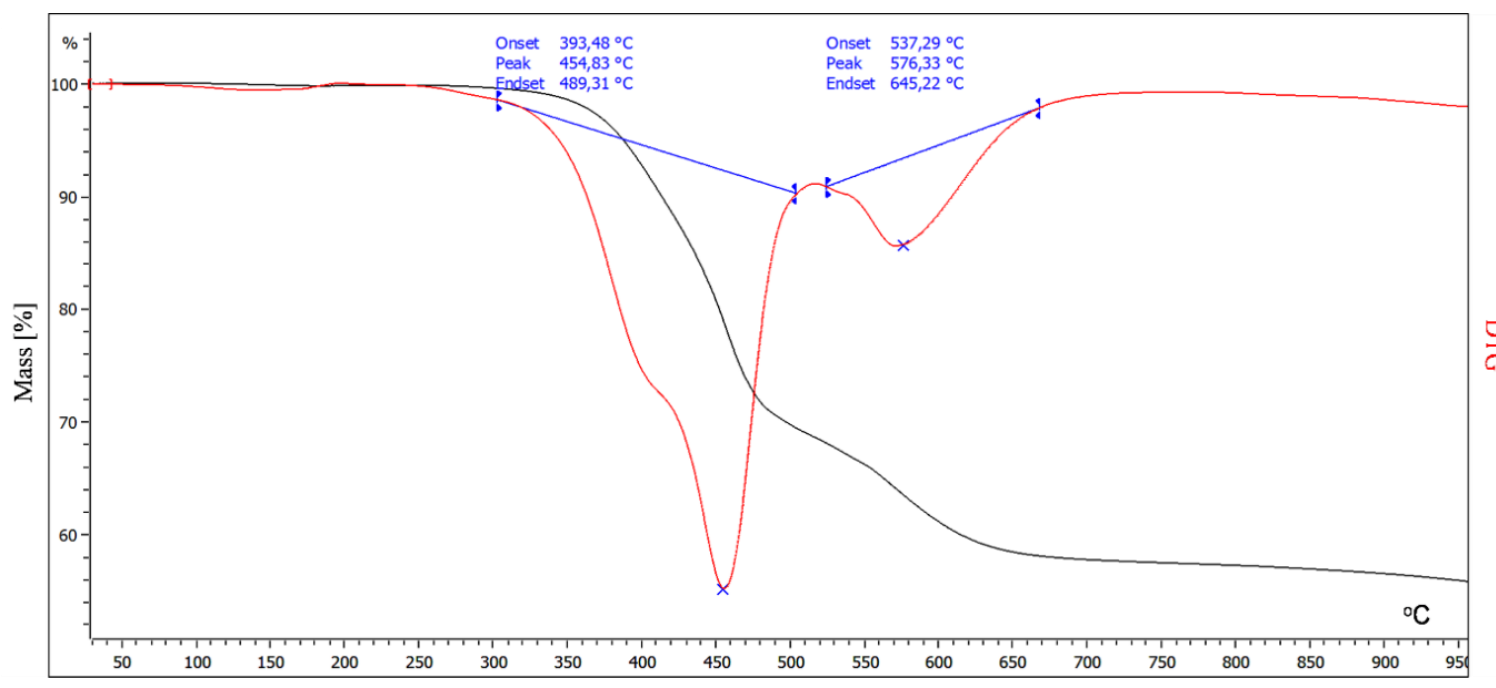

Figure S19. DTG curves of DDSQa in nitrogen atmosphere.

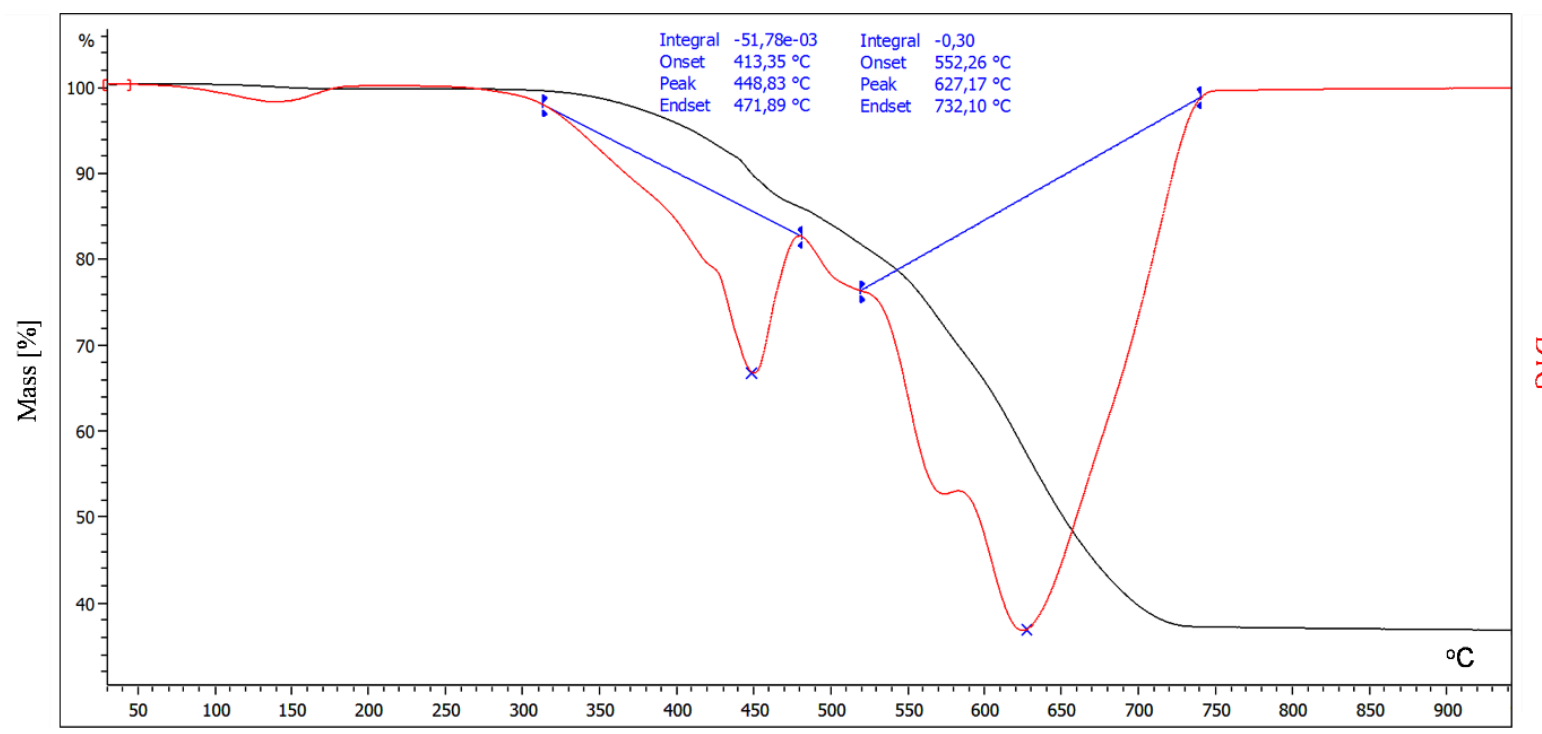

Figure S20. DTG curves of DDSQb in air atmosphere. 


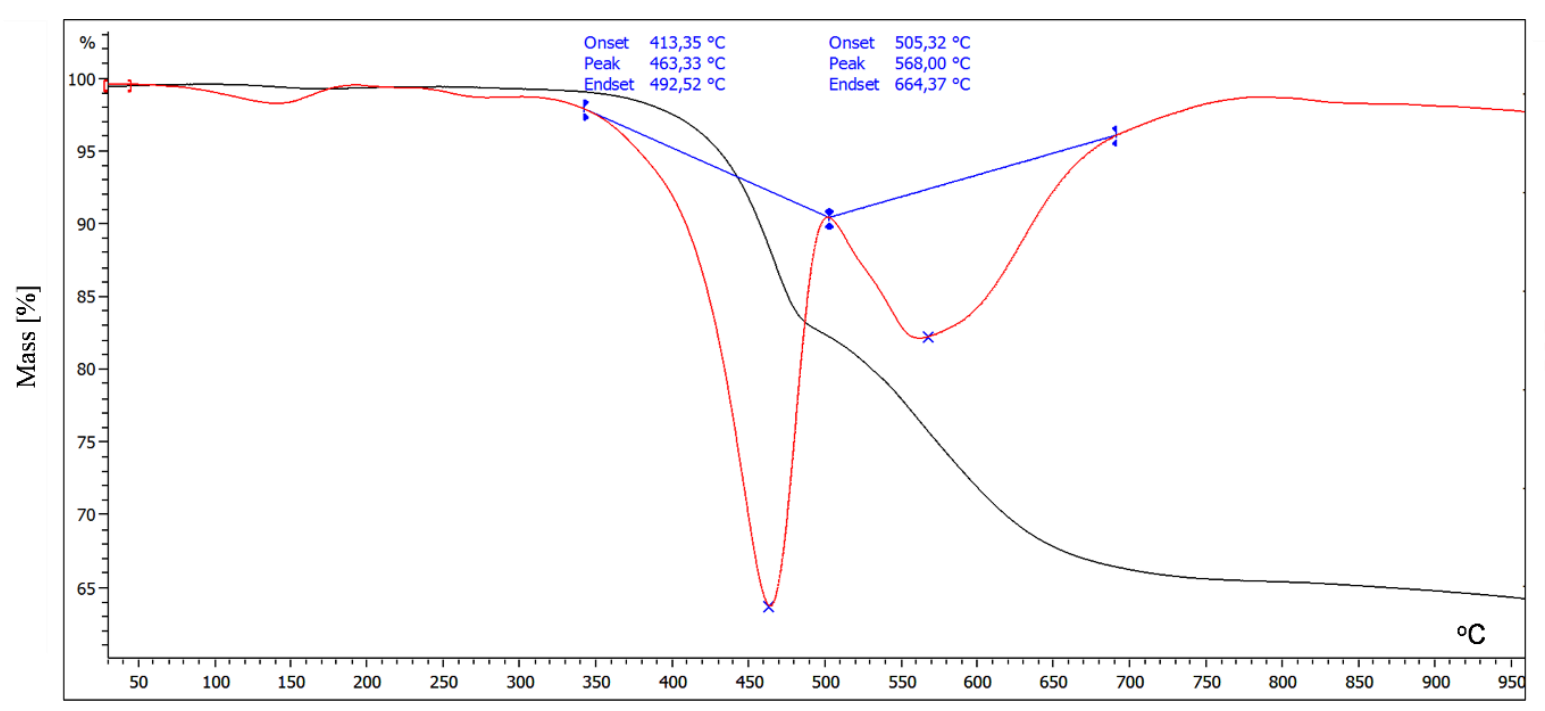

Figure S21. DTG curves of DDSQb in nitrogen atmosphere.

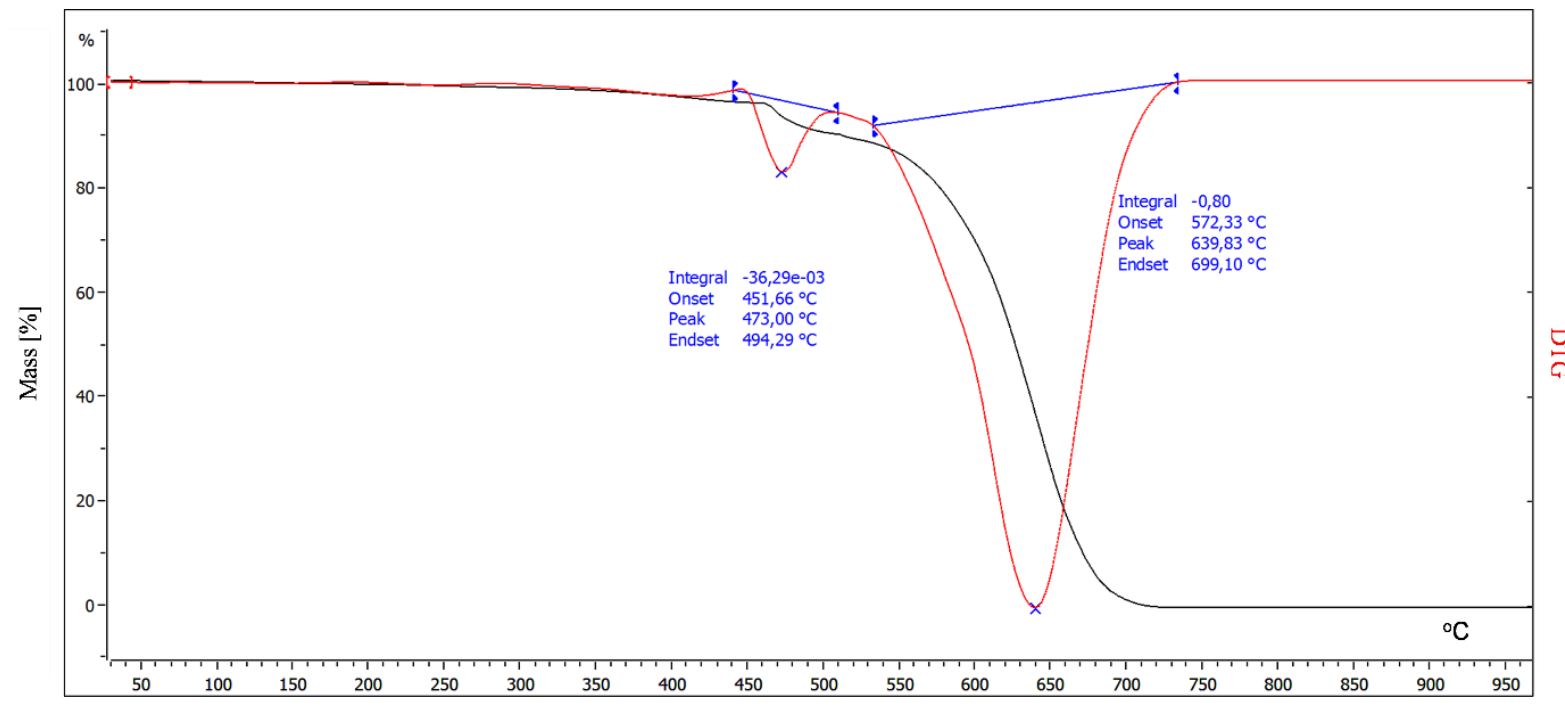

Figure S22. DTG curves of $\mathbf{T}$ in air atmosphere.

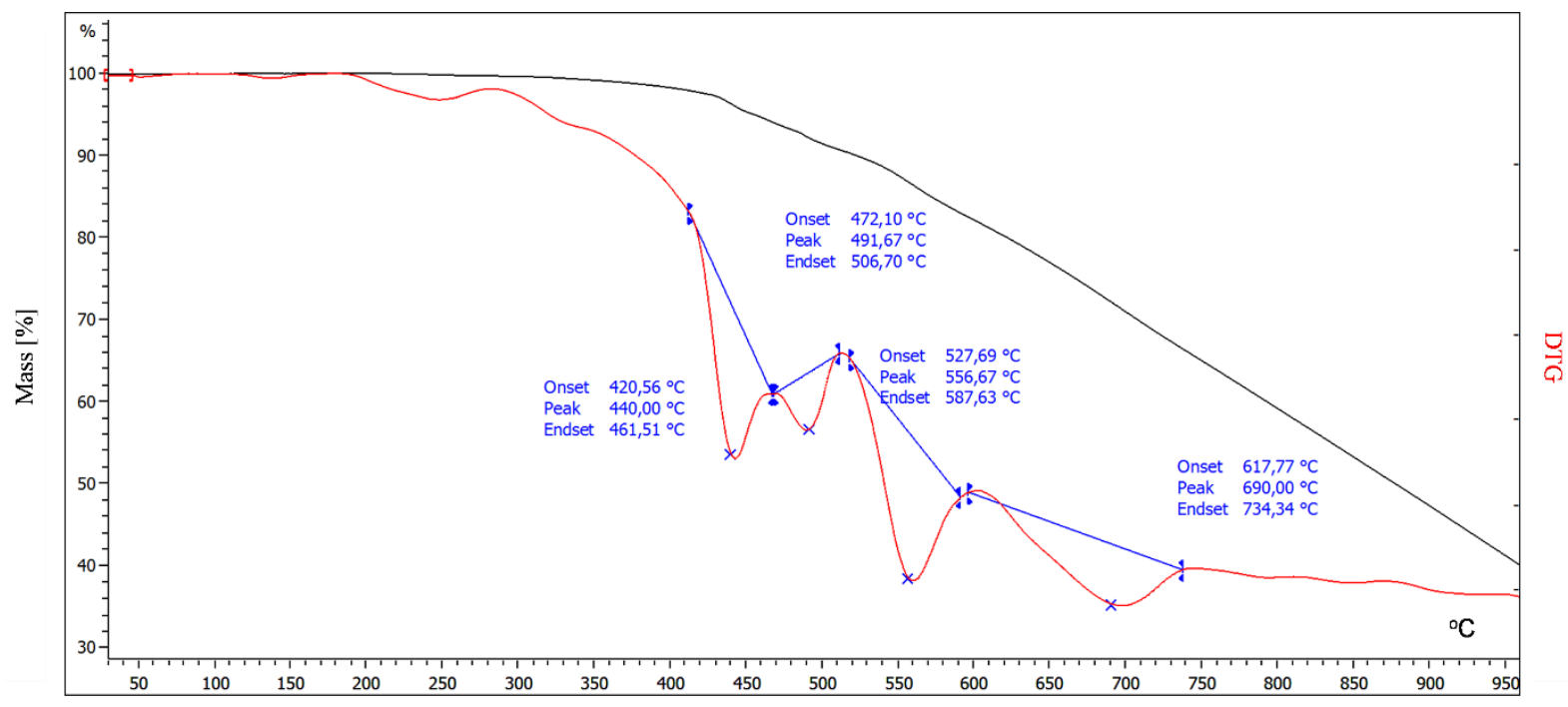

Figure S23. DTG curves of $\mathbf{T}$ in nitrogen atmosphere. 


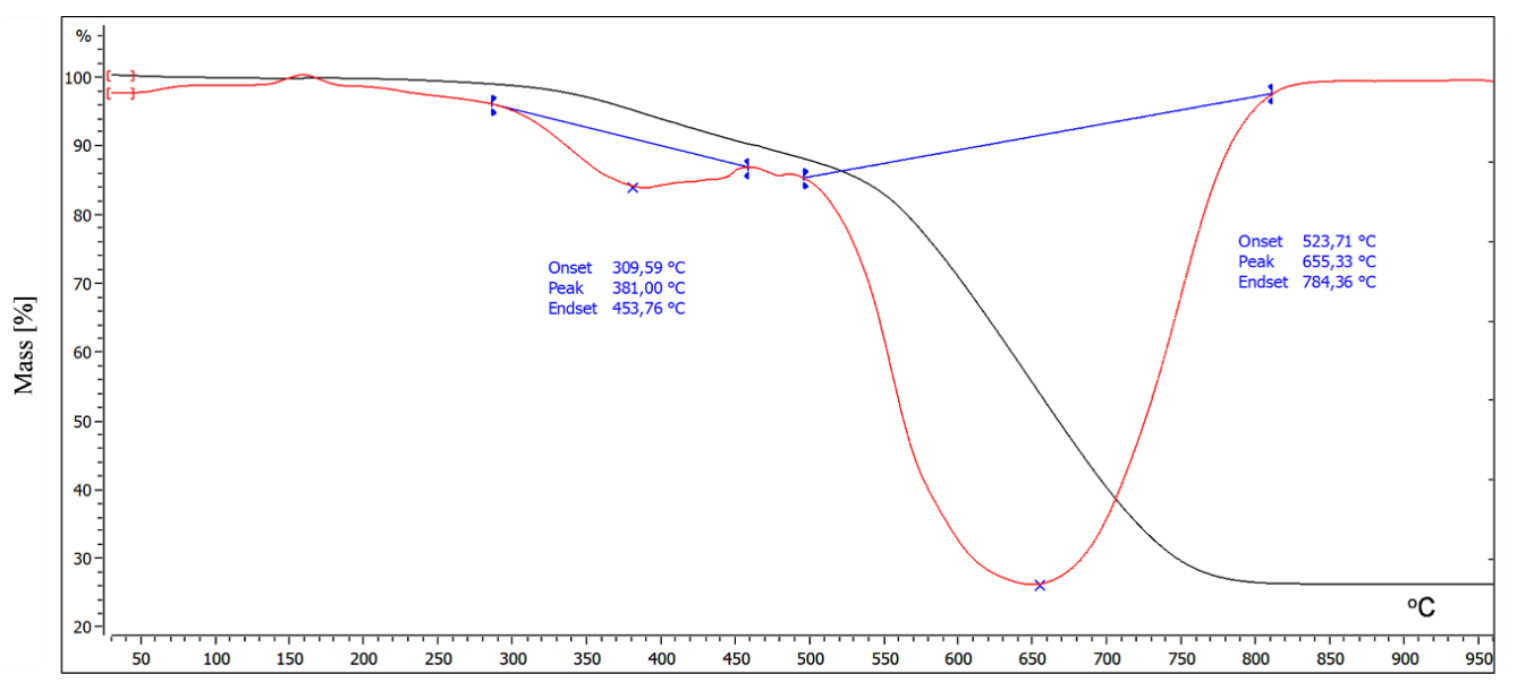

Figure S24. DTG curves of DDSQ_Ta in air atmosphere.

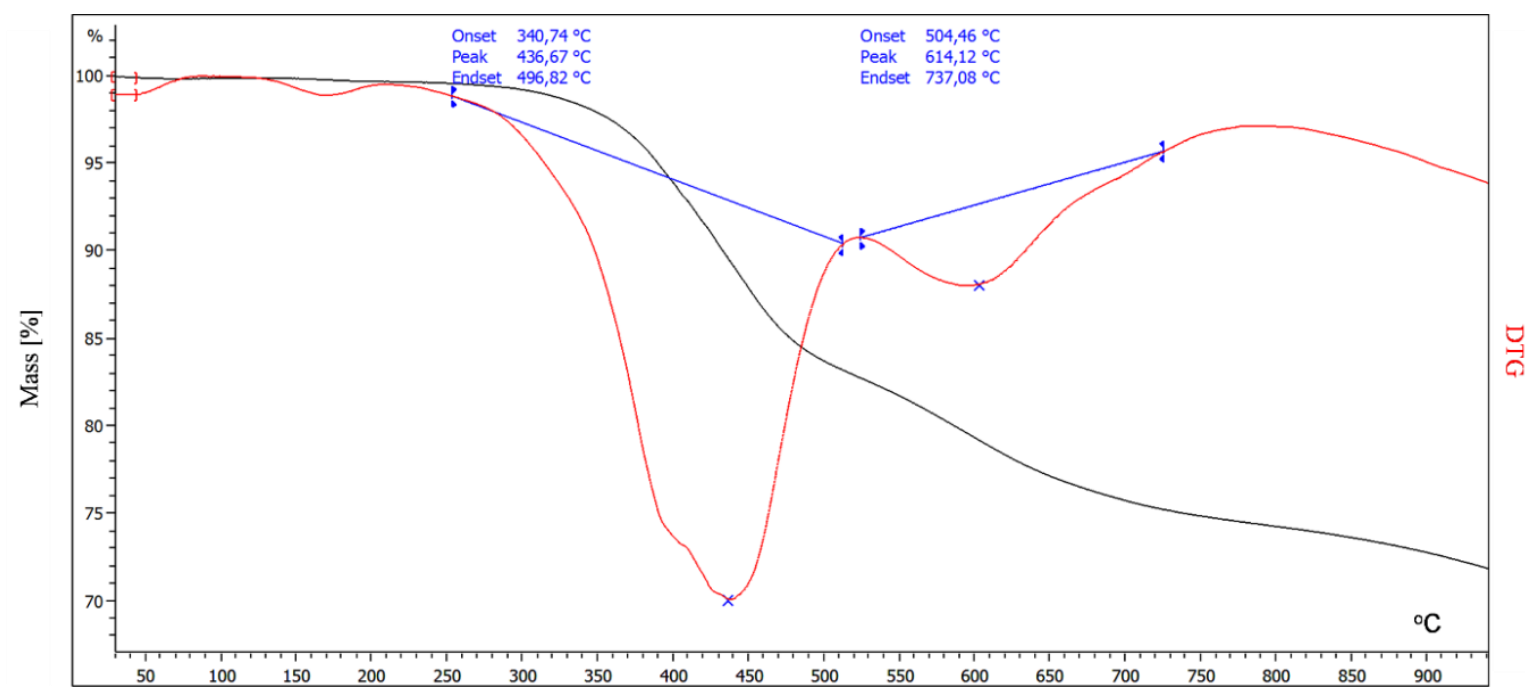

Figure S25. DTG curves of DDSQ_Ta in nitrogen atmosphere.

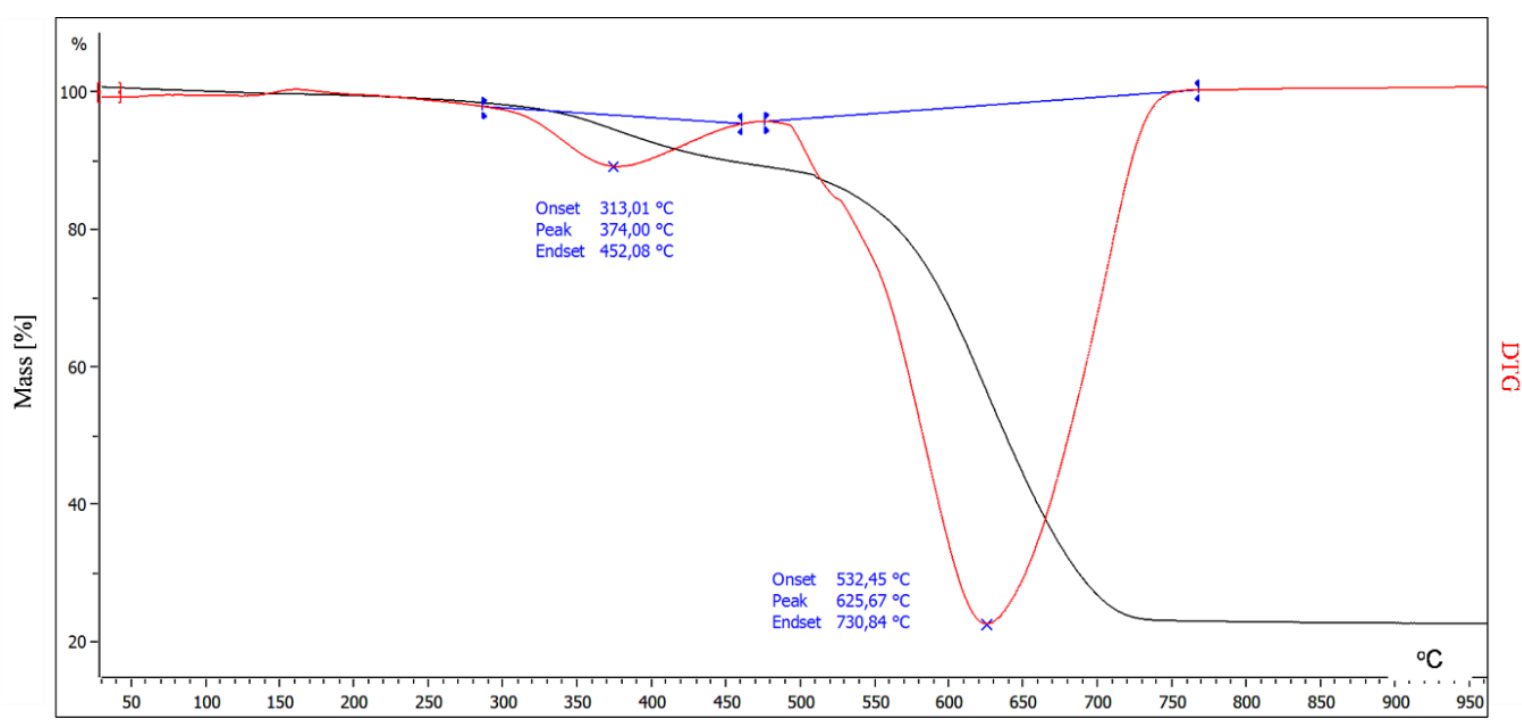

Figure S26. DTG curves of DDSQ_Tb in air atmosphere. 


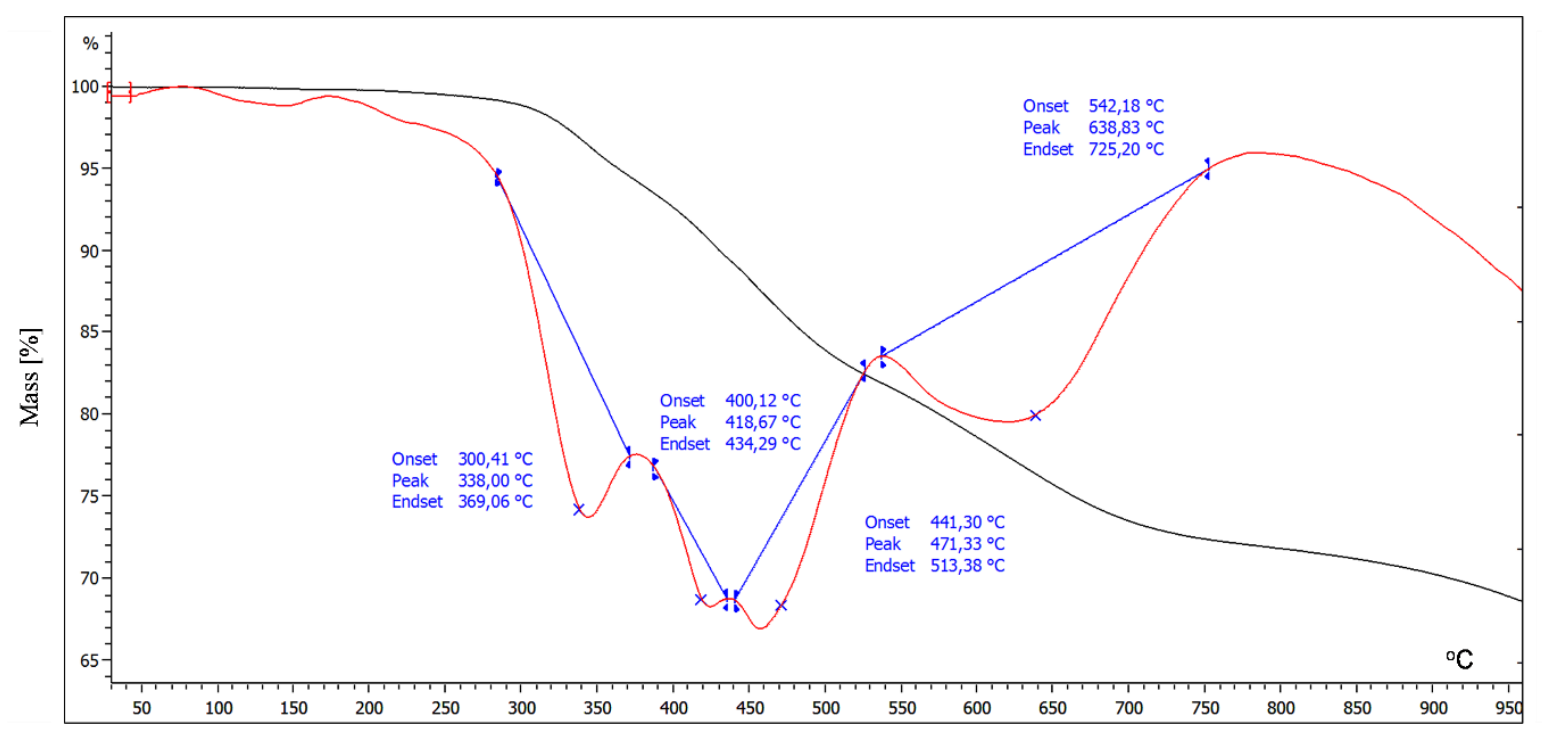

$\stackrel{\square}{a}$

Figure S27. DTG curves of DDSQ_Tb in nitrogen atmosphere.

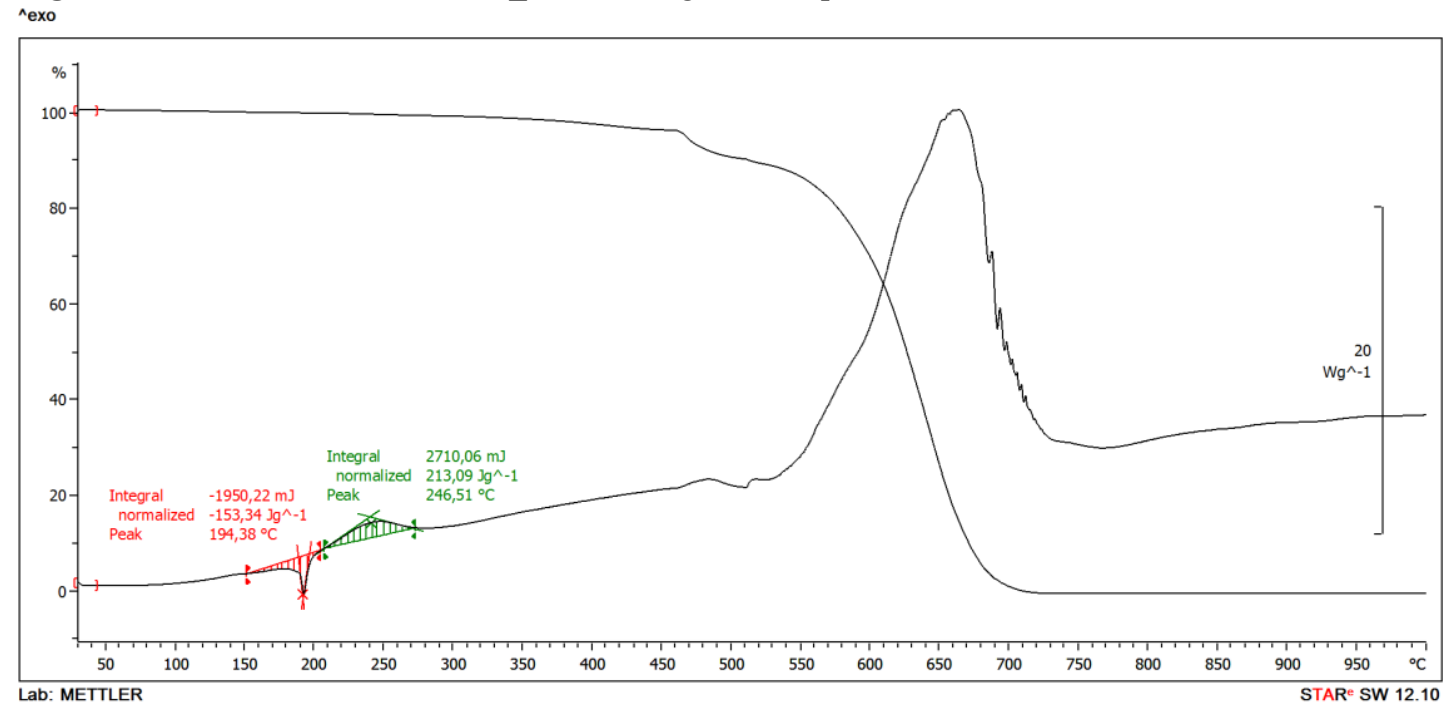

Figure S28. TGA/DSC curves of $\mathbf{T}$ in air atmosphere.

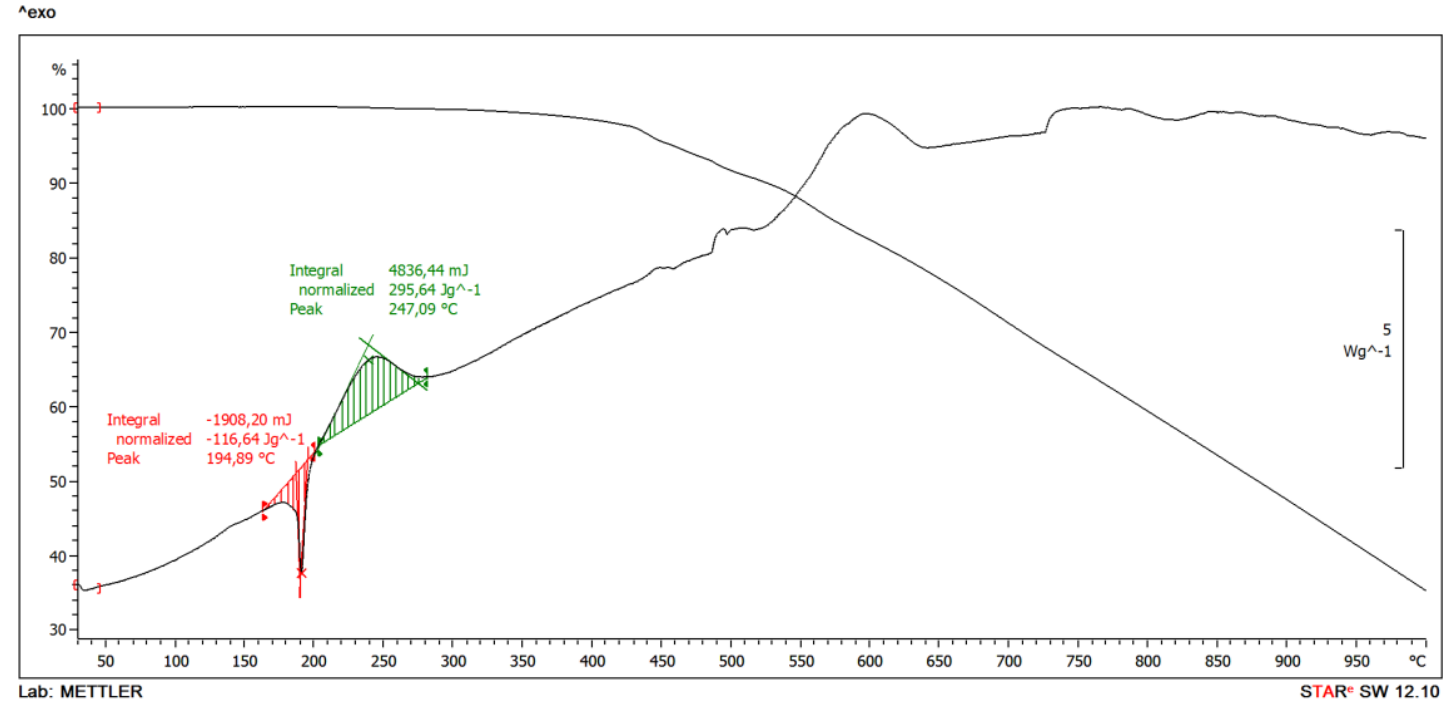

Figure S29. TGA/DSC curves of $\mathbf{T}$ in nitrogen atmosphere. 


\section{Results of absorption - emission analysis of DDSQa-b and DDSQ_Ta-b}

a)

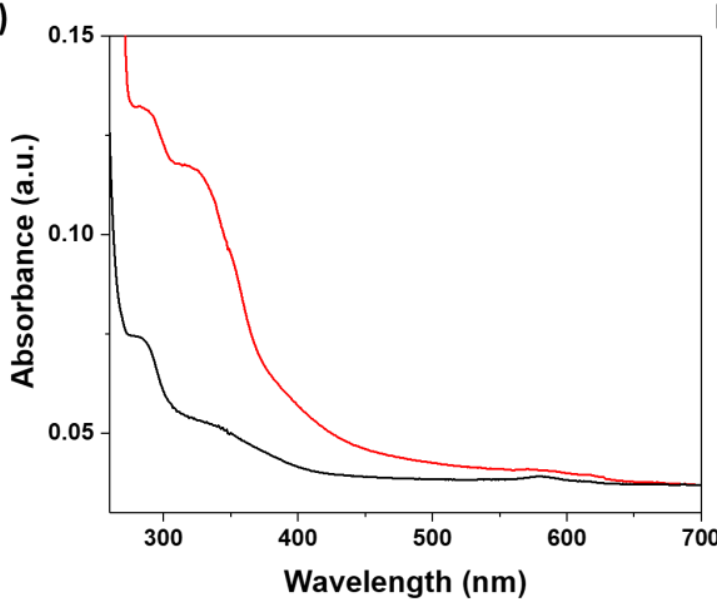

b)

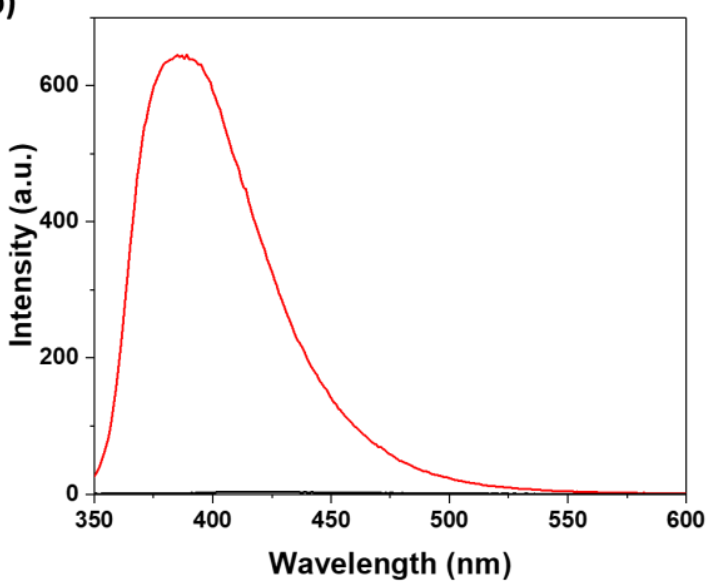

Figure S30. (a) Absorption and (b) emission spectra of the sample DDSQa (black) and the DDSQ_Ta (red) in $\mathrm{CH}_{2} \mathrm{Cl}_{2}\left(1 \times 10^{-6} \mathrm{M}\right) . \lambda_{\mathrm{ex}}=310 \mathrm{~nm}$, Slits $=5 \mathrm{~nm}$.

a)

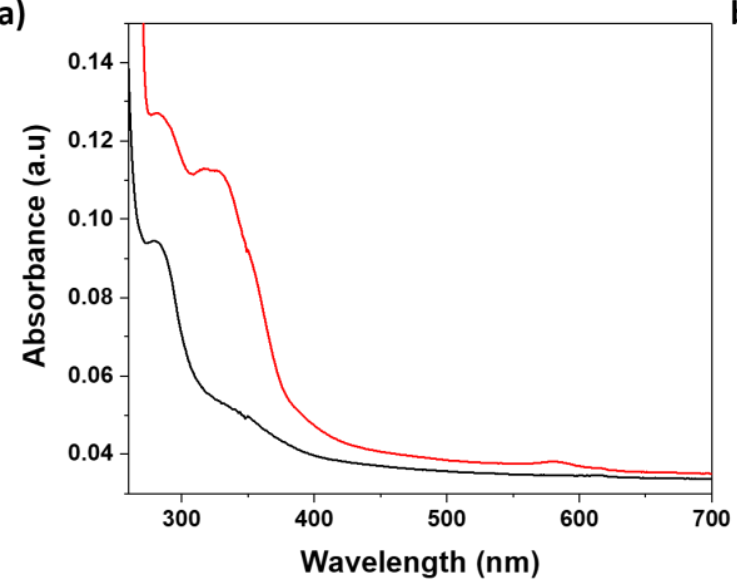

b)

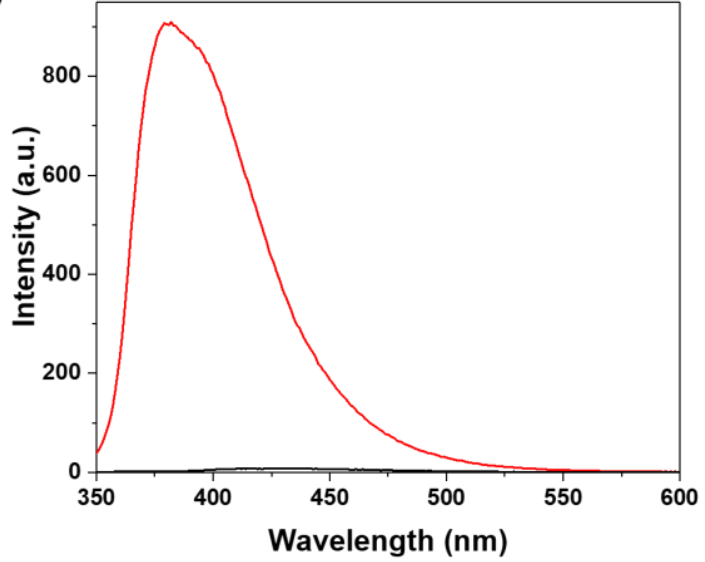

Figure S31. (a) Absorption and (b) emission spectra of the comparison of the compound DDSQb (black) and the DDSQ_Tb (red) in $\mathrm{CH}_{2} \mathrm{Cl}_{2}\left(1 \times 10^{-6} \mathrm{M}\right) . \lambda_{\mathrm{ex}}=310 \mathrm{~nm}$, Slits $=5 \mathrm{~nm}$. 
a)

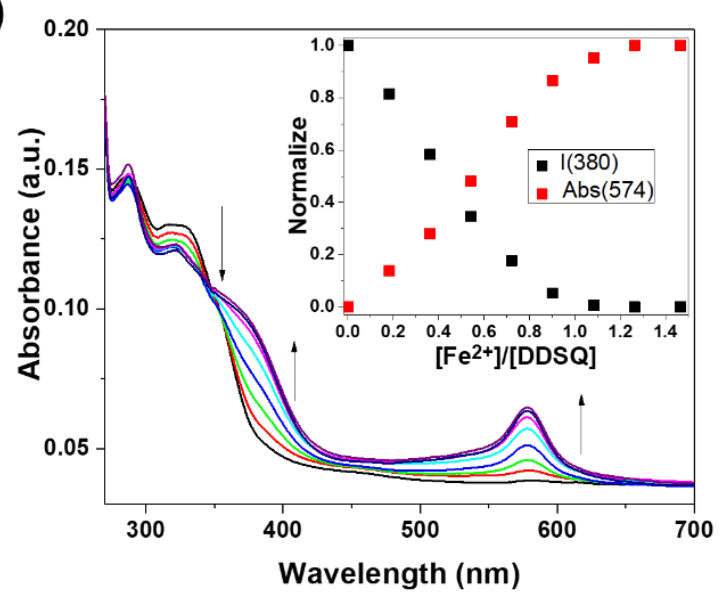

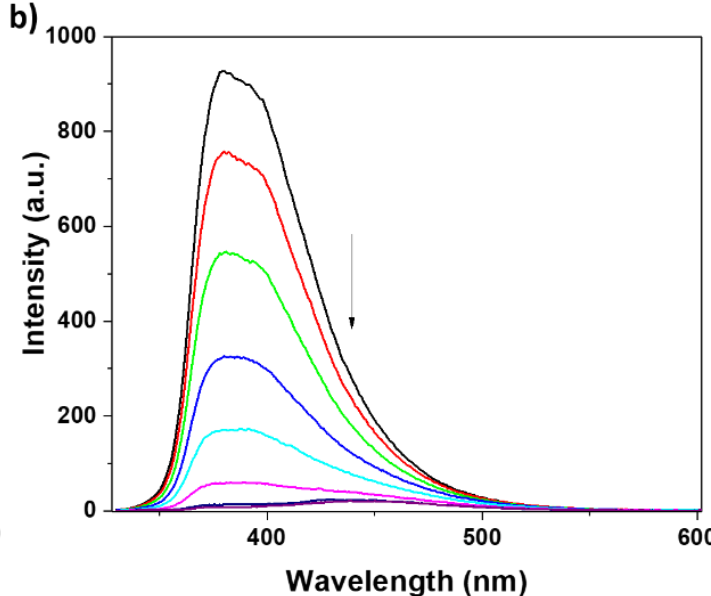

Figure S32. (a) UV-vis Absorption spectra of the sample DDSQ_Tb in $\mathrm{CH}_{2} \mathrm{Cl}_{2}\left(1 \times 10^{-6} \mathrm{M}\right)$ upon titration with $\mathrm{Fe}(\mathrm{OTf})_{2}$ in $\mathrm{EtOH}\left(3.63 \times 10^{-4} \mathrm{M}\right)$. The inset shows the normalized absorption changes at $574 \mathrm{~nm}$ (red squares) and the normalized emission intensity changes at $380 \mathrm{~nm}$ (black squares). (b) Emission spectra of the compound DDSQ_Tb in $\mathrm{CH}_{2} \mathrm{Cl}_{2}\left(1 \times 10^{-6} \mathrm{M}\right)$ upon titration with $\mathrm{Fe}(\mathrm{OTf})_{2}$ in EtOH $\left(3.63 \times 10^{-4} \mathrm{M}\right) . \lambda_{\text {ex }}=310 \mathrm{~nm}$, and $\mathrm{OD}=$ 0.13 , Slits $=5 \mathrm{~nm}$.

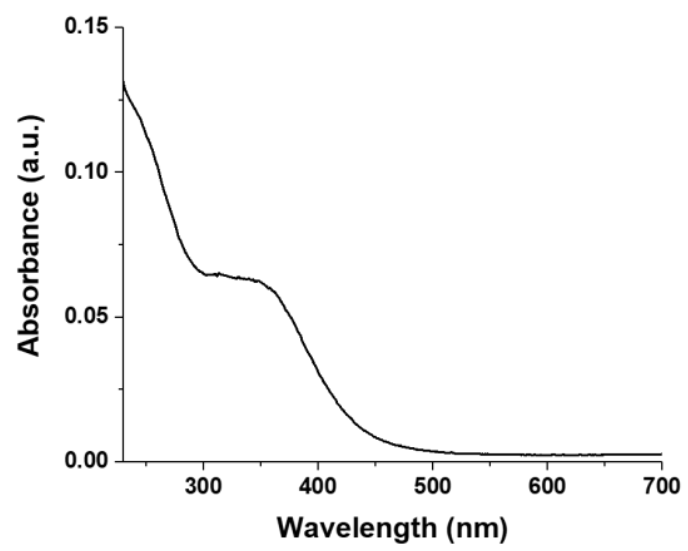

Figure S33. UV-vis Absorption spectra of $\mathrm{Fe}(\mathrm{OTf})_{2}$ in $\mathrm{EtOH}\left(3.63 \times 10^{-4} \mathrm{M}\right)$.

a)

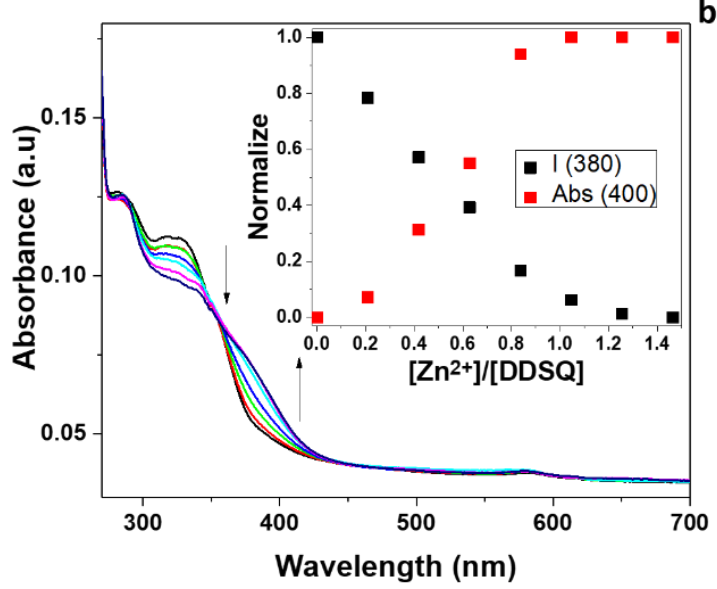

b)

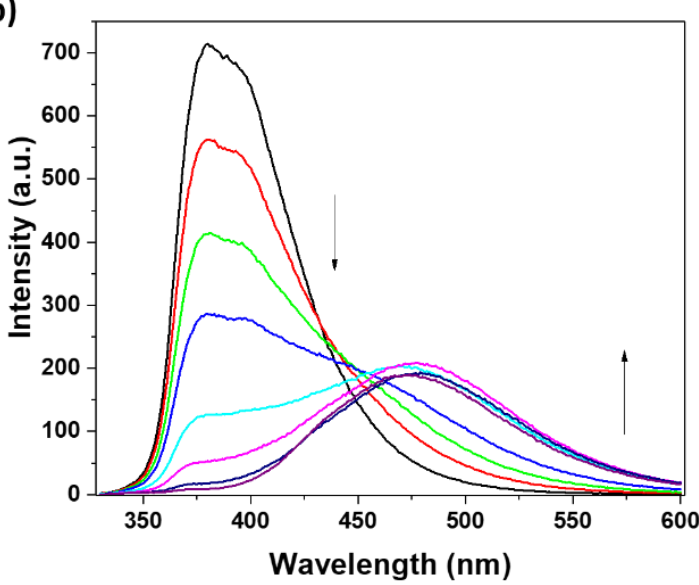

Figure S34. (a) UV-vis Absorption spectra of sample DDSQ_Tb in $\mathrm{CH}_{2} \mathrm{Cl}_{2}\left(1 \times 10^{-6} \mathrm{M}\right)$ upon titration with $\mathrm{Zn}(\mathrm{OTf})_{2}$ in $\mathrm{EtOH}\left(4.18 \times 10^{-4} \mathrm{M}\right)$. The inset shows the normalized absorption changes at $400 \mathrm{~nm}$ (red squares) and the normalized emission intensity changes at $380 \mathrm{~nm}$ (black squares). (b) Emission spectra of the compound DDSQ_Tb in $\mathrm{CH}_{2} \mathrm{Cl}_{2}\left(1 \times 10^{-6} \mathrm{M}\right)$ upon titration with $\mathrm{Zn}(\mathrm{OTf})_{2}$ in EtOH $\left(4.18 \times 10^{-4} \mathrm{M}\right) . \lambda_{\mathrm{ex}}=310 \mathrm{~nm}$, and $\mathrm{OD}=$ 0.12 , Slits $=5 \mathrm{~nm}$. 


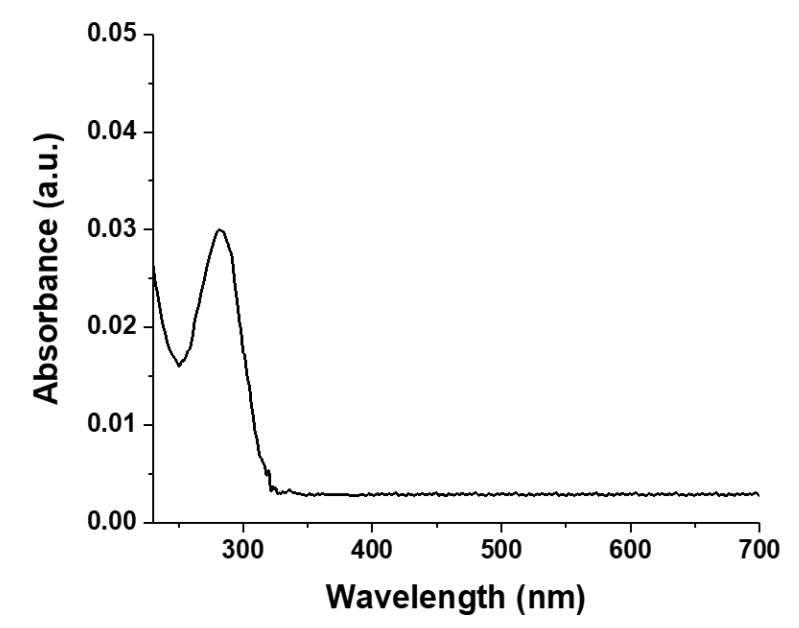

Figure S35. UV-vis Absorption spectra of $\mathrm{Zn}(\mathrm{OTf})_{2}$ in $\mathrm{EtOH}\left(4.18 \times 10^{-4} \mathrm{M}\right)$.

a)

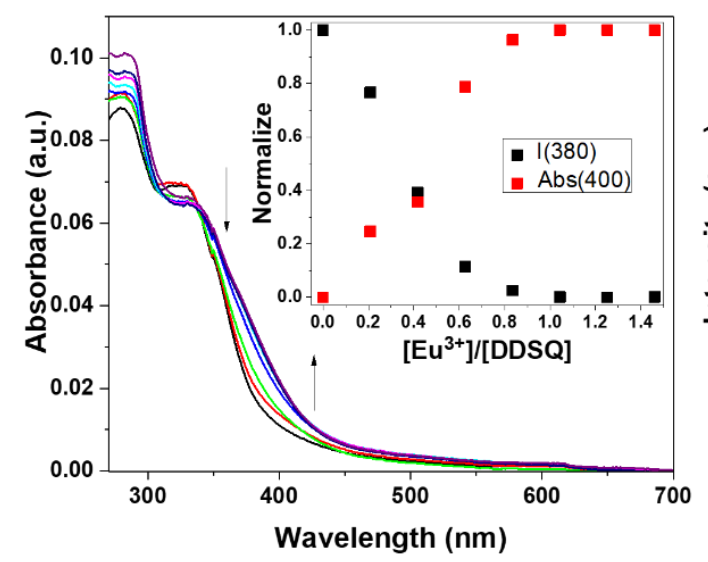

b)

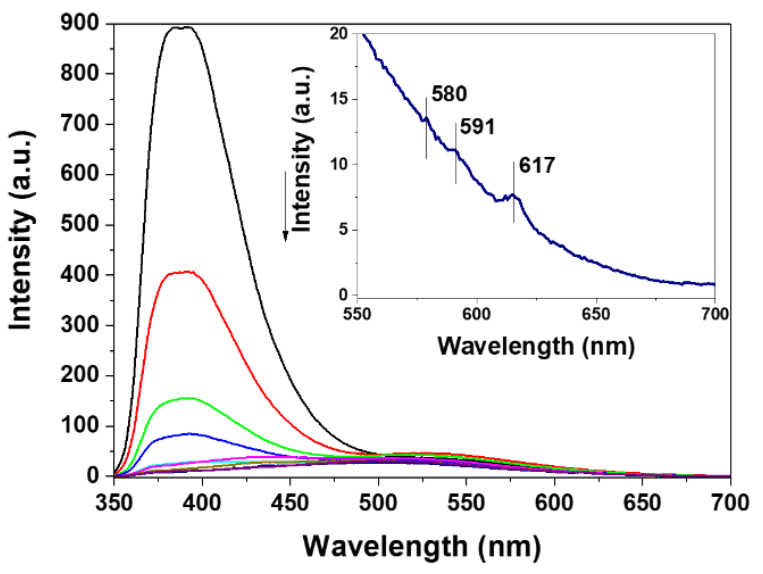

Figure S36. (a) UV-vis Absorption spectra of the compound DDSQ_Tb in $\mathrm{CH}_{2} \mathrm{Cl}_{2}\left(1 \times 10^{-6} \mathrm{M}\right)$ upon titration with $\mathrm{Eu}(\mathrm{OTf})_{3}$ in $\mathrm{EtOH}\left(4.17 \times 10^{-4} \mathrm{M}\right)$. The inset shows the normalized absorption changes at $400 \mathrm{~nm}$ (red squares) and the normalized emission intensity changes at $380 \mathrm{~nm}$ (black squares). (b) Emission spectra of the compound DDSQ_Tb in $\mathrm{CH}_{2} \mathrm{Cl}_{2}\left(1 \times 10^{-6} \mathrm{M}\right)$ upon titration with $\mathrm{Eu}(\mathrm{OTf})_{3}$ in $\mathrm{EtOH}\left(4.17 \times 10^{-4} \mathrm{M}\right)$. The inset shows the emission spectra in the range of $550-700 \mathrm{~nm}$. $\lambda_{\mathrm{ex}}=310 \mathrm{~nm}$, and $\mathrm{OD}=0.08$, Slits $=5 \mathrm{~nm}$.

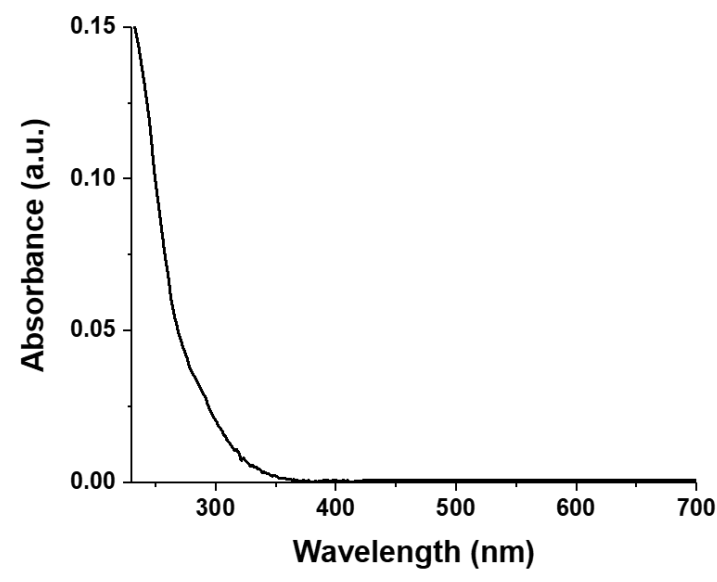

Figure S37. UV-vis Absorption spectra of $\mathrm{Eu}(\mathrm{OTf})_{3}$ in $\mathrm{EtOH}\left(4 \cdot 17 \times 10^{-4} \mathrm{M}\right)$. 

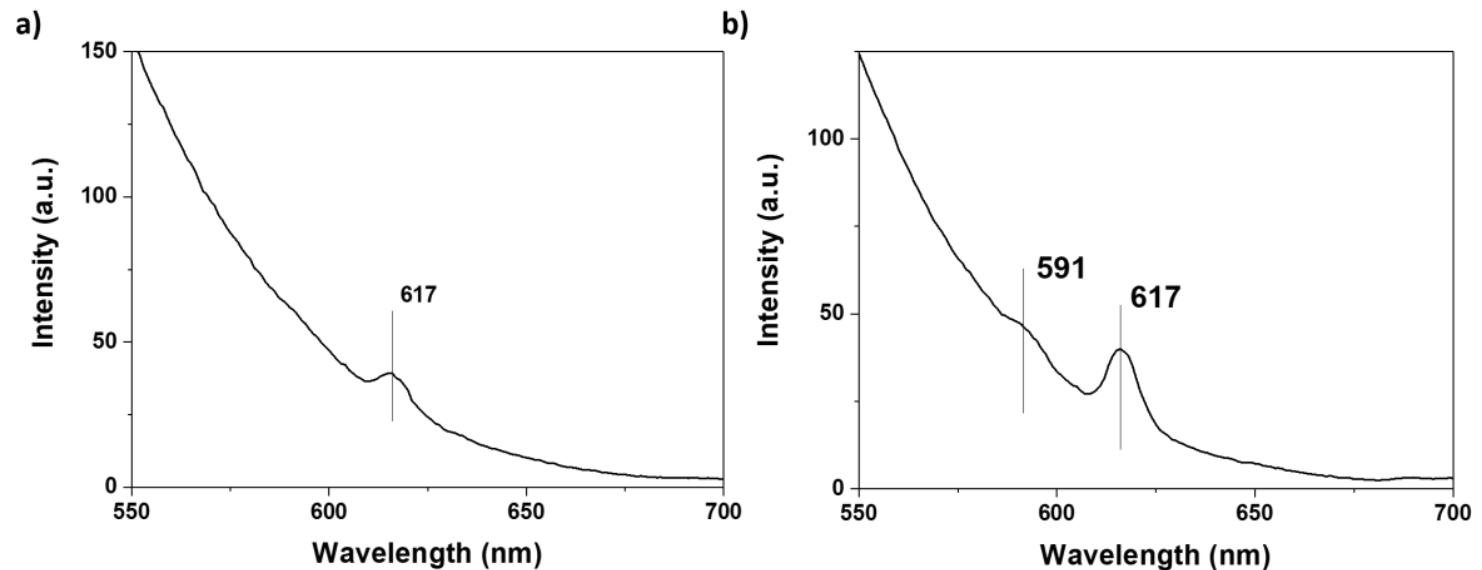

Figure S38. Typical Europium line like emission centred at $617 \mathrm{~nm}$ clearly visible in (a) compound DDSQ_Ta in $\mathrm{CH}_{2} \mathrm{Cl}_{2} / \mathrm{CH}_{3} \mathrm{CN}(3 / 97)\left(1 \times 10^{-6} \mathrm{M}\right)$ upon titration with $\mathrm{Eu}(\mathrm{OTf})_{3}$ in $\mathrm{EtOH}\left(4.17 \times 10^{-4} \mathrm{M}\right)$. (b) And compound DDSQ_Tb in $\mathrm{CH}_{2} \mathrm{Cl}_{2} / \mathrm{CH}_{3} \mathrm{CN}(3 / 97)\left(1 \times 10^{-6} \mathrm{M}\right)$ upon titration with Eu(OTf $)_{3}$ in EtOH $\left(4.17 \times 10^{-4} \mathrm{M}\right)$. Range of $550-700 \mathrm{~nm} . \lambda_{\mathrm{ex}}=310 \mathrm{~nm}$, Slits $=5 \mathrm{~nm}$.

a)

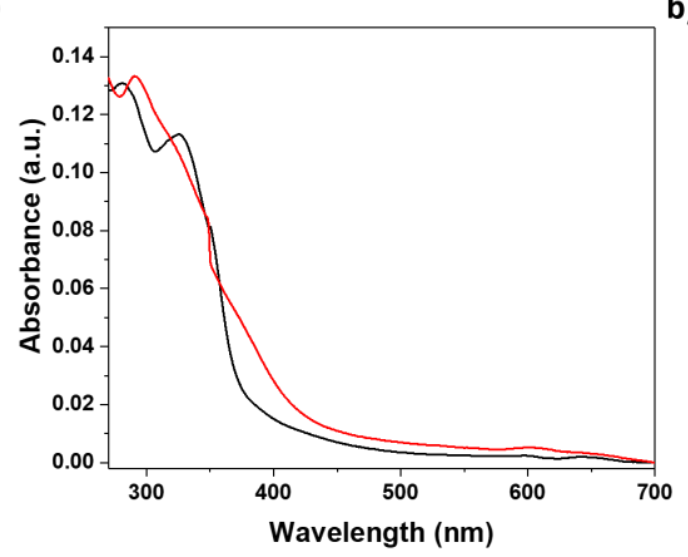

b)

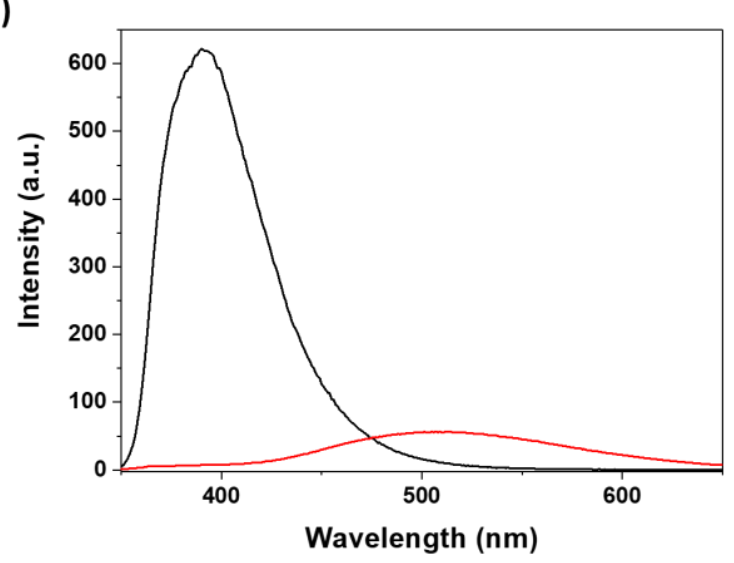

Figure S39. Absorption spectra and emission spectra of $E$ (black line) to $Z$ (red line) isomerization of the sample DDSQ_Ta. $\lambda_{\mathrm{ex}}=310 \mathrm{~nm}$, Slits $=5 \mathrm{~nm}$.

a)

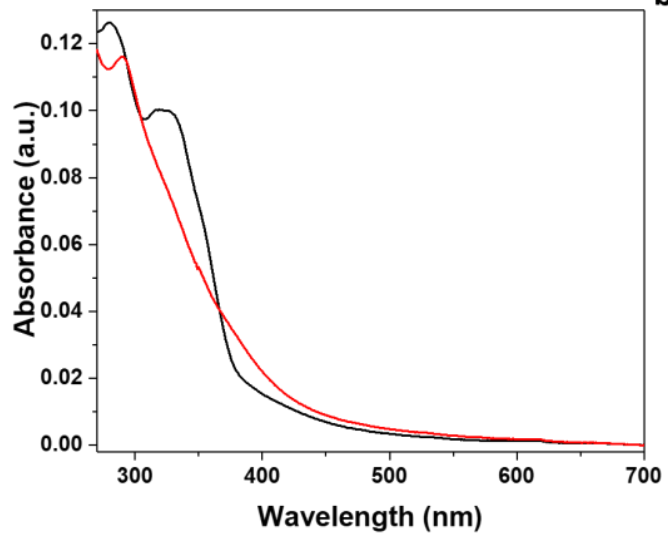

b)

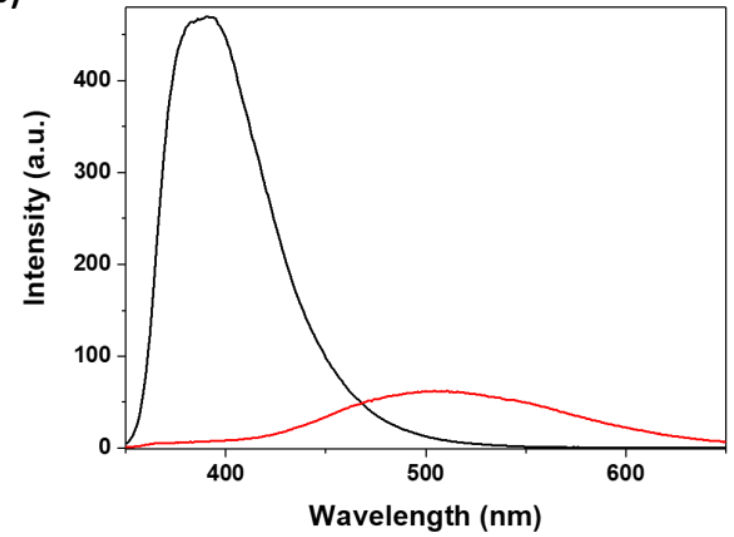

Figure S40. Absorption spectra and emission spectra of $E$ (black line) to $Z$ (red line) isomerization of the sample DDSQ_Tb. $\lambda_{\text {ex }}=310 \mathrm{~nm}$, Slits $=5 \mathrm{~nm}$. 
a)

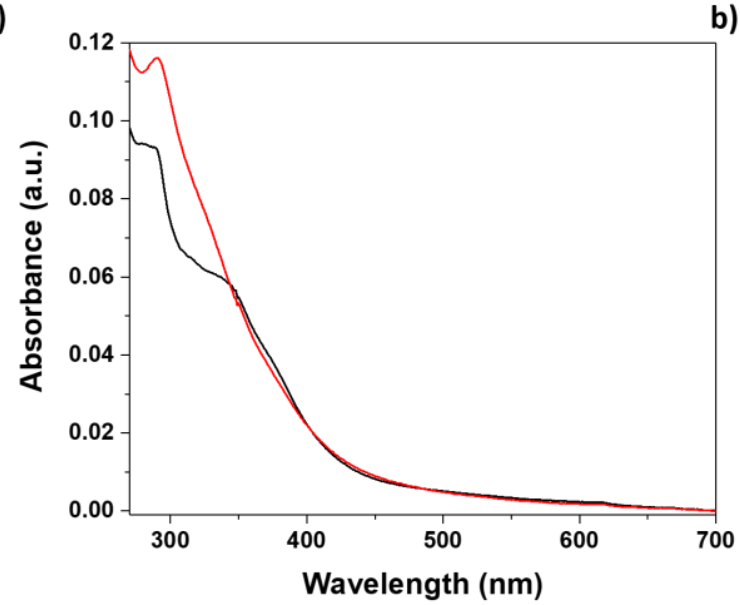

b)

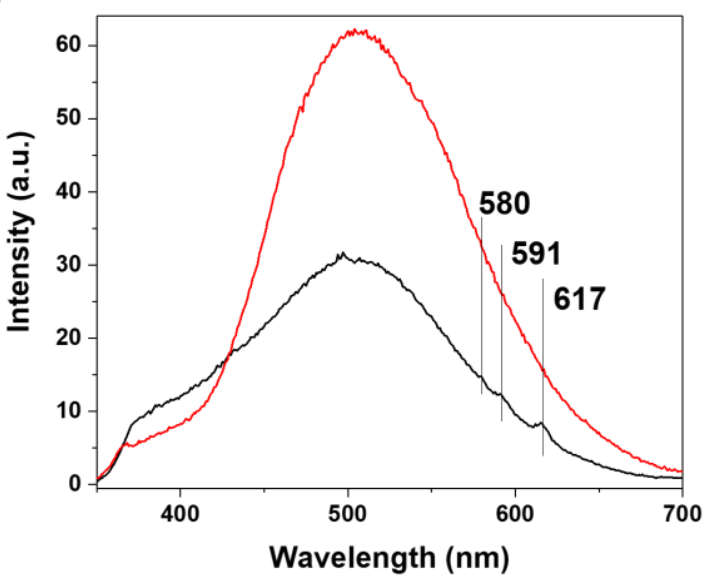

Figure S41. Absorption spectra and emission spectra of Eu@DDSQ_Tb $E$ (black line) and $Z$ (red line) isomers. $\lambda_{\mathrm{ex}}=310 \mathrm{~nm}$, Slits $=5 \mathrm{~nm}$.

a)

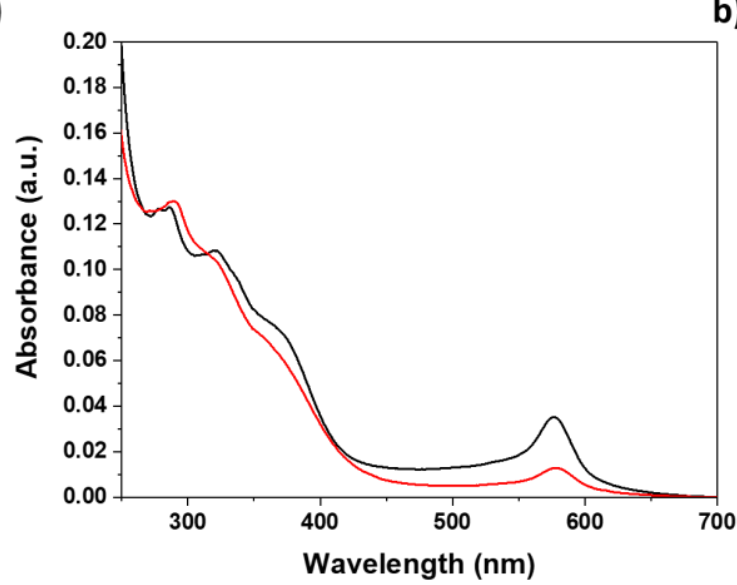

b)

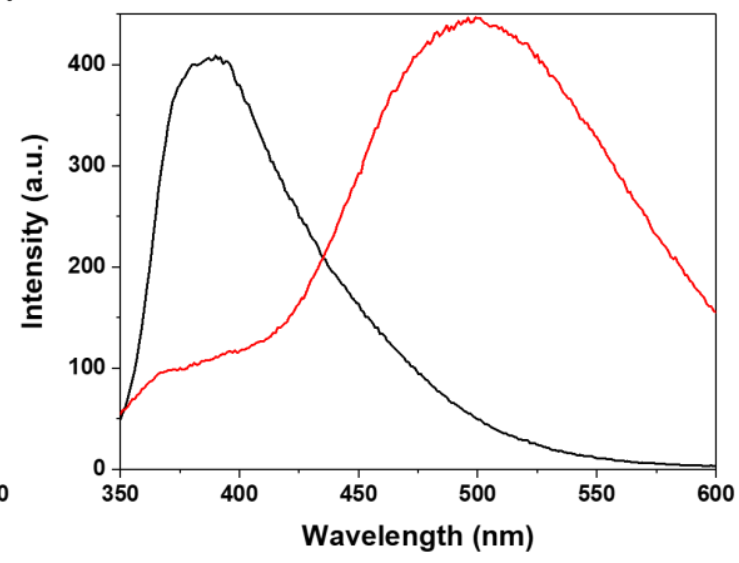

Figure S42. Absorption spectra and emission spectra of Fe@DDSQ_Ta $E$ (black line) and $Z$ (red line) isomers. $\lambda_{\mathrm{ex}}=310 \mathrm{~nm}$, Slits $=5 \mathrm{~nm}$.
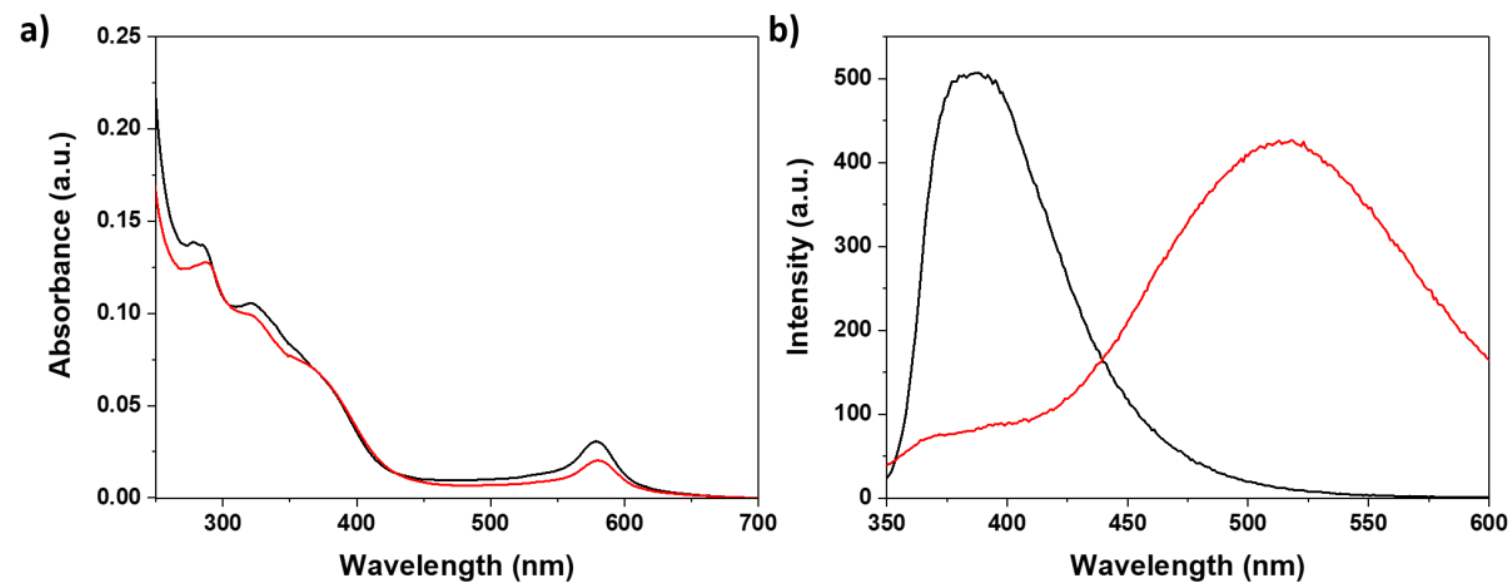

Figure S43. Absorption spectra and emission spectra of Fe@DDSQ_Tb E (black line) and Z (red line) isomers. $\lambda_{\mathrm{ex}}=310 \mathrm{~nm}$, Slits $=5 \mathrm{~nm}$. 

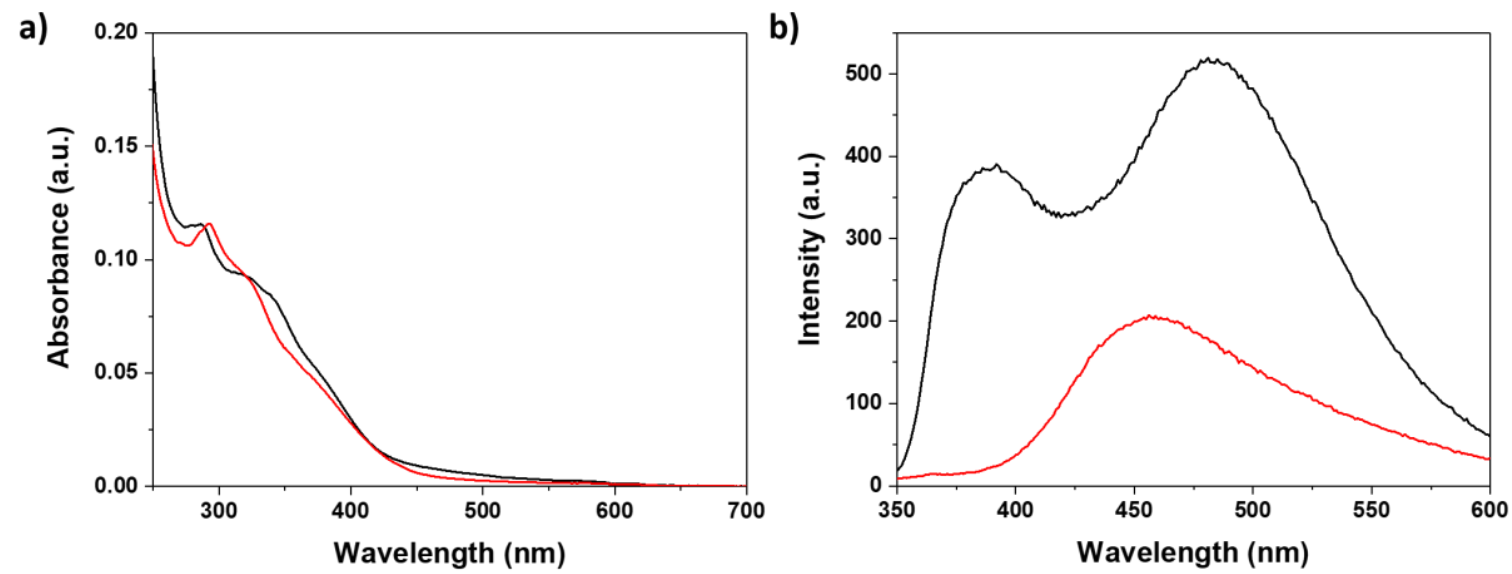

Figure S44. Absorption spectra and emission spectra of Zn@DDSQ_Ta $E$ (black line) and $Z$ (red line) isomers. $\lambda_{\mathrm{ex}}=310 \mathrm{~nm}$, Slits $=5 \mathrm{~nm}$.
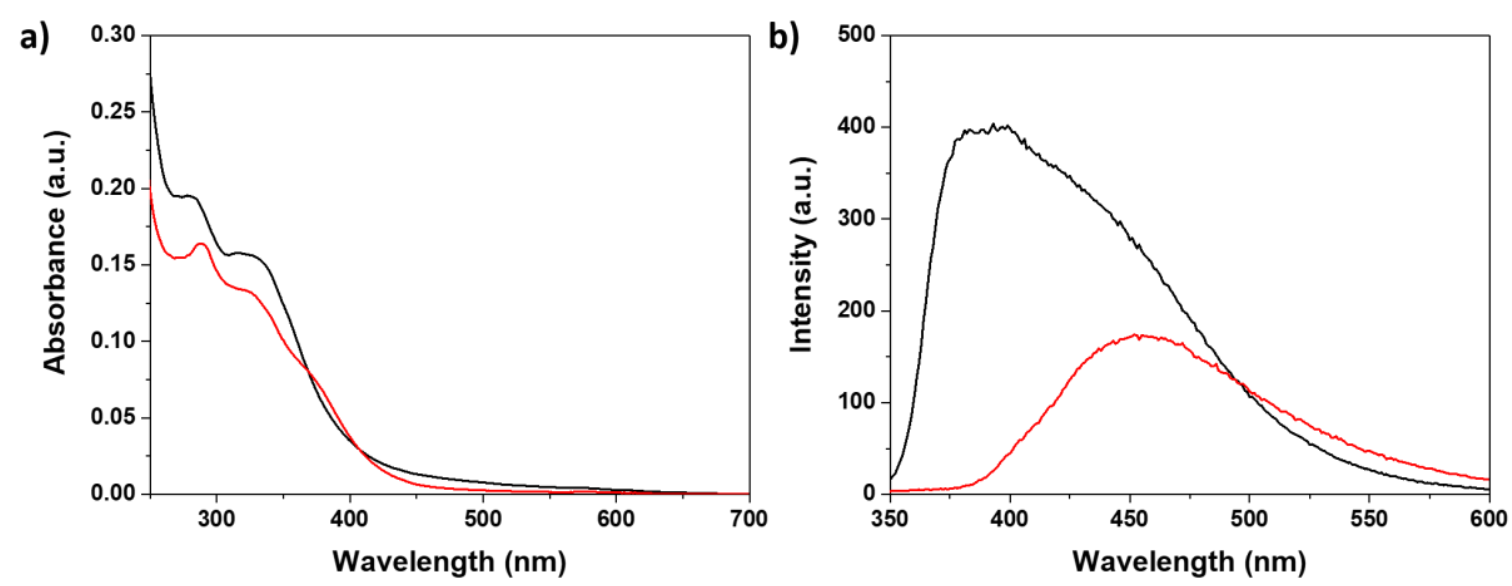

Figure S45. Absorption spectra and emission spectra of Zn@DDSQ_Tb $E$ (black line) and Z (red line) isomers. $\lambda_{\mathrm{ex}}=310 \mathrm{~nm}$, Slits $=5 \mathrm{~nm}$. 
5. Results of analysis of DDSQa-b and DDSQ_Ta-b
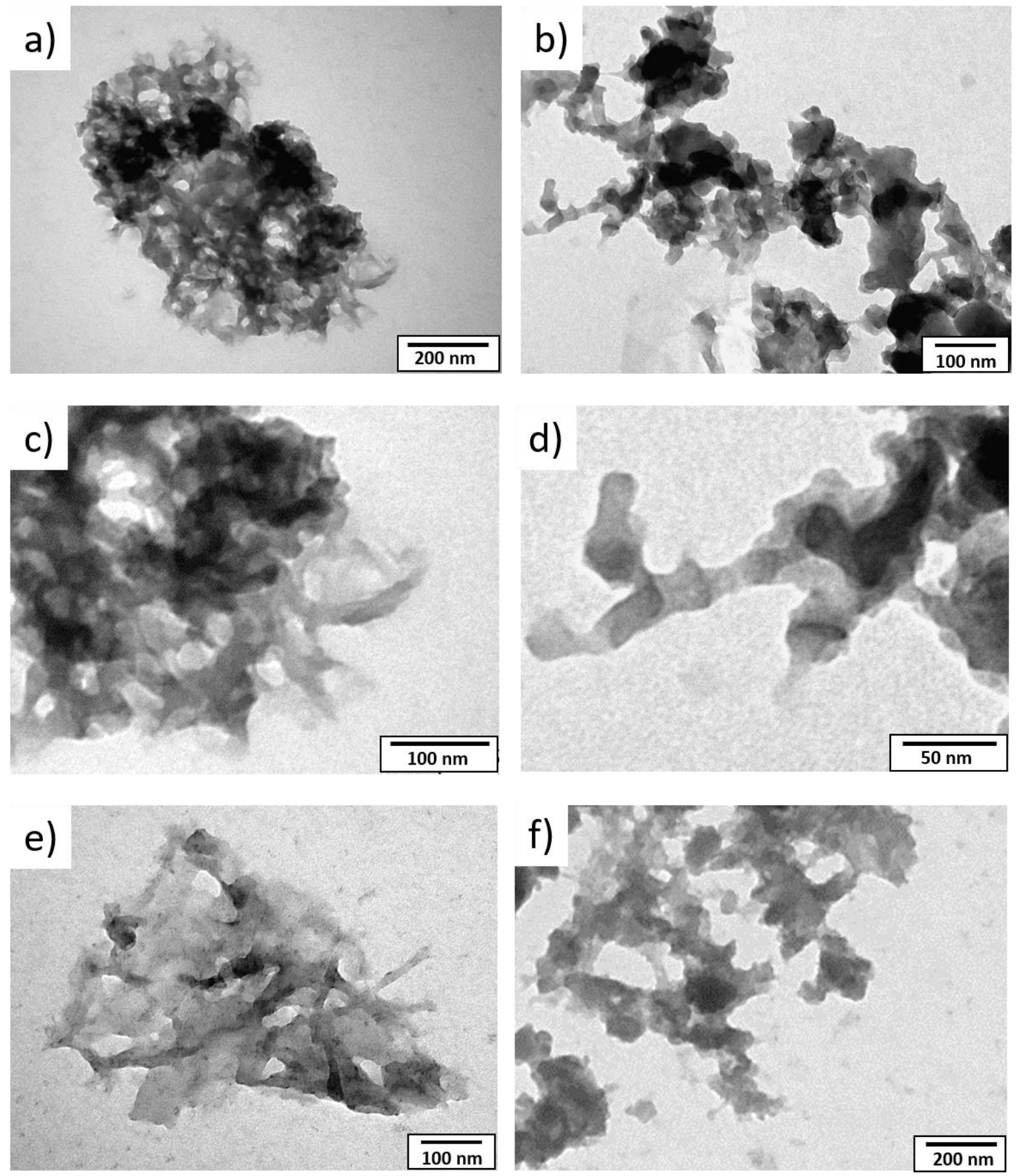

Figure S46. Transmission Electron Microscopy images of the lyophilized a) and c) Zn@DDSQ_Ta, b) and d) Zn@DDSQ_Tb, e) 2Eu@3DDSQ_Ta and f) 2Eu@3DDSQ_Tb. 

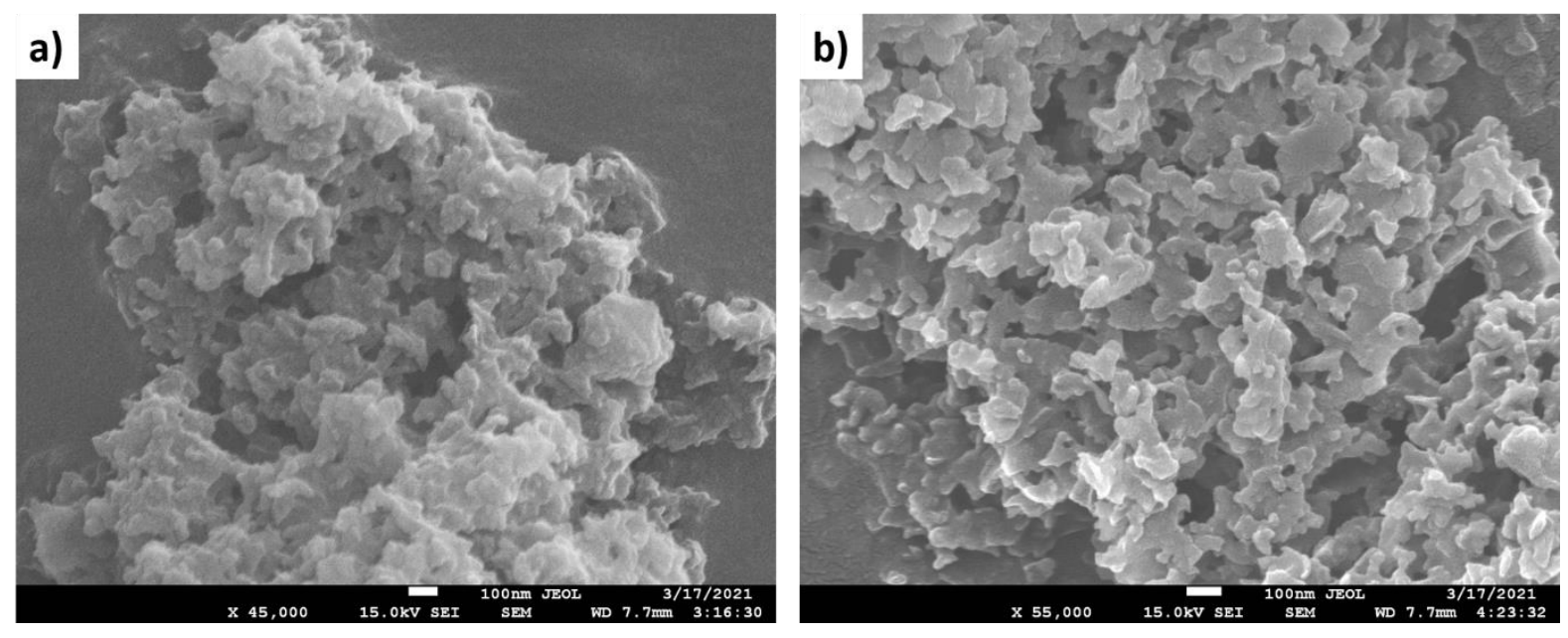

Figure S47. Scanning Electron Microscopy (SEM) images of the lyophilized (a) Fe@DDSQ_Ta and (b) Fe@DDSQ_Tb. 


\section{References}

1 Winter, A.; Egbe, D. A. M.; Schubert, U. S. Rigid $\pi$-Conjugated Mono-, Bis-, and Tris(2,2':6',2"-terpyridines). Org. Lett. 2007, 9, 2344-2348.

2 Żak, P.; Dudziec, B.; Kubicki, M.; Marciniec, B. Silylative Coupling versus Metathesis-Efficient Methods for the Synthesis of Difunctionalized Double-Decker Silsesquioxane Derivatives. Chem. - A Eur. J. 2014, 20, 9387-9393. 